INSTITUT NATIONAL DE RECHERCHE EN INFORMATIQUE ET EN AUTOMATIQUE

\title{
A Newton method using exact jacobians for solving fluid-structure coupling
}

\author{
Miguel Ángel Fernández — Marwan Moubachir
}

$\mathbf{N}^{\circ} \mathbf{5 0 8 7}$

THÈME 4

\section{apport}

derecherche 



\title{
A Newton method using exact jacobians for solving fluid-structure coupling
}

\author{
Miguel Ángel Fernández*, Marwan Moubachir ${ }^{\dagger}$ \\ Thème 4 - Simulation et optimisation \\ de systèmes complexes \\ Projet OPALE \\ Rapport de recherche $n^{\circ} 5087-32$ pages
}

\begin{abstract}
This report aims at introducing a partitioned Newton based method for solving nonlinear coupled systems arising in the numerical approximation of fluid-structure interaction problems. We provide a method which characteristic lies in the use of exact cross jacobians evaluation involving the shape derivative of the fluid state with respect to solid motion perturbations. Numerical tests based on an implementation inside a 3D fluid-structure interaction code show how the exactness of the cross jacobians computation guarantee the overall convergence of the Newton's loop.
\end{abstract}

Key-words: Fluid-Struture interaction, Navier-Stokes equations, ALE formulation, full coupled schemes, Newton methods, shape sensitivity analysis, haemodynamics.

\footnotetext{
* École Polytechnique Fédérale de Lausanne, IACS, 1015 Lausanne, Switzerland, email:miguel.fernandez@inria.fr

† INRIA Sophia-Antipolis - Projet OPALE / E-lab OpRaTel, email:Marwan.Moubachir@inria.fr
} 


\section{Une méthode de Newton utilisant l'évaluation exacte des jacobiennes pour la résolution de problèmes couplés fluide-structure}

Résumé : Dans ce rapport de recherche, nous présentons une méthode de Newton partitionnée afin de résoudre les systèmes couplés nonlinéaires issus de l'approximation numérique de problèmes d'interaction fluide-structure. La caractéristique principale de la méthode réside dans l'évaluation exacte des jacobiennes croisées faisant intervenir la dérivée de forme de l'état fluide vis-à-vis de perturbations des mouvements du solide. Nous montrons, grâce à des tests numériques fondés sur une implémentation au sein d'un code d'interaction fluidestructure $3 \mathrm{D}$, comment l'exactitude de l'évaluation des jacobiennes croisées permet de garantir la convergence globale de la boucle de Newton.

Mots-clés : Interaction fluide-structure, équations de Navier-Stokes, formulation ALE, schémas totalement couplés, méthodes de Newton, analyse de sensibilité de forme, hémodynamique. 


\section{Introduction}

Nowadays, Computational Fluid-Structure Dynamics (CFSD) spreads out in every engineering field, from aeroelasticity to bio-mechanics problems (see, for instance, $[26,8,10,22,14$, $25,23,29,16,31])$. One issue arising in the numerical approximation of these nonlinear coupled systems, is the definition of coupling algorithms based on specific solvers involving efficient discretization for each of the solid and fluid subsystems, that may guarantee accurate and fast convergence of the overall system. This issue is particularly difficult to face when the structure is light, namely, when the fluid and the solid densities are of the same order, as it happens in haemodynamics for example. Indeed, in this case, numerical experiments show that only fully coupled schemes can ensure stability of the resulting method (see $[22,28,6,16,23,24]$ ). Thus, at each time step, the rule is to solve a coupled highly non-linear system using efficient methods that may preserve, inside inner loops, the fluid-structure subsystem splitting.

Standard and simple strategies to solve these non-linear problems are fixed-point based methods $[3,1]$. Unfortunately, these methods are very expensive (even if several acceleration techniques may improve their efficiency [25,6]) and may fail to converge [16]. Recent advances in this topic suggest the use of Newton based methods for a fast convergence towards the solution of the non-linear coupled system $[1,23,24,31,16,19]$. These methods rely on the evaluation of the jacobians associated to the fluid-solid coupled state equations. More precisely, the critical step consists in the evaluation of the cross jacobians [31], e.g. the sensitivity of the fluid state with respect to solid motions. Up to now, this difficulties have been overcome either by using finite difference approximations $[23,24,31,19]$, or by replacing the tangent operator of the coupled system by a simpler operator $[31,16,5,7,17]$. In both cases, such approximations may deteriorate or avoid the overall convergence [31]. In this paper, we provide an explicit expression of these cross jacobians, using shape sensitivity calculus [30], in the case of an incompressible Newtonian fluid coupled with a nonlinear elastic solid under large displacements. These expressions are then implemented inside a 3D finite element (FE) library and we show on some model cases the superiority of using exact jacobians against approximated versions of the Newton's method.

The reminder of the paper is organized as follows. In section 2, we introduce a general fluid-solid coupled system and describe its associated mathematical model. In section 3 , the resulting coupled set of equations is used to build a coupled weak variational formulation. The latter is discretized in time using a fully coupled scheme in section 4 , where, the resulting nonlinear coupled system is turned into an abstract form. In order to solve this abstract coupled system, in section 5 we describe the main steps of the Newton's method in terms of fluid and solid operators and its derivatives. The expressions of such derivatives are obtained, in section 6 , using general shape derivative calculus results that are recalled in appendix $\mathrm{A}$. These expressions have been already announced in $[12,13]$ as brief notes. In section 7 , we detail the major steps of the Newton's algorithm applied to the coupled fluidsolid problem. The resulting algorithm has been implemented in a 3D fluid-solid research code. The numerical results are presented in section 8 , showing the relevance of our approach. Finally, we give some conclusions and draw some lines for future works.

$\mathrm{RR} \quad \mathrm{n}^{\circ} 5087$ 


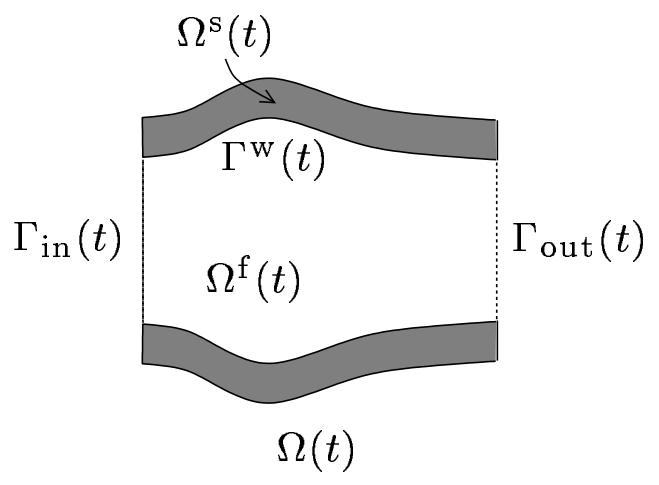

Figure 1: Geometric configurations

\section{Mechanical problem}

Let us first describe a general non-linear fluid-structure system in large displacements. We consider a mechanical system occupying a moving domain $\Omega(t)$. It consists of a deformable structure $\Omega^{\mathrm{s}}(t)$ (vessel wall, pipe-line, ...) surrounding a fluid under motion (blood, oil, $\ldots$ ) in the complement $\Omega^{\mathrm{f}}(t)$ of $\Omega^{\mathrm{s}}(t)$ in $\Omega(t)$ (see figure 1 ). The problem is to determinate the time evolution of the configuration $\Omega(t)$, as well as the velocity and Cauchy stress tensor within the fluid and the structure. The latter being governed by the classic conservation laws of the continuum mechanics, endowed with appropriate constitutive laws.

The evolution of $\Omega(t)$ (see e.g. [8, 20,22]), can be described through a smooth and injective mapping

$$
\mathbf{x}: \Omega_{0} \times \mathbb{R}^{+} \longrightarrow \mathbb{R}^{3},
$$

which maps any point $\mathbf{x}_{0}$ of a given fixed (reference) configuration $\Omega_{0} \stackrel{\text { def }}{=} \Omega_{0}^{\mathrm{f}} \cup \Omega_{0}^{\mathrm{s}}$ into its corresponding image $\mathbf{x}\left(\mathbf{x}_{0}, t\right)$, inside the present configuration $\Omega(t)$ (see figure 2). We set $\mathbf{x}^{\mathrm{f}} \stackrel{\text { def }}{=} \mathbf{x}_{\mid \Omega_{0}^{\mathrm{f}}}$ and $\mathbf{x}^{\mathrm{s}} \stackrel{\text { def }}{=} \mathbf{x}_{\mid \Omega_{0}^{\mathrm{s}}}$. For $\mathbf{x}_{0} \in \Omega_{0}^{\mathrm{s}}, \mathbf{x}^{\mathrm{s}}\left(\mathbf{x}_{0}, t\right)$ represents the position at time $t \geq 0$ of the material point $\mathbf{x}_{0}$ inside the solid domain. This corresponds to the classical lagrangian flow. In particular, the solid displacement $\mathbf{d}^{\mathrm{s}}\left(\mathbf{x}_{0}, t\right)$ reads

$$
\mathbf{d}^{\mathrm{s}}\left(\mathbf{x}_{0}, t\right) \stackrel{\text { def }}{=} \mathbf{x}^{\mathrm{s}}\left(\mathbf{x}_{0}, t\right)-\mathbf{x}_{0}, \quad \mathbf{x}_{0} \in \Omega_{0}^{\mathrm{s}} .
$$

This implies that the configuration velocity matches the solid velocity inside the solid domain. Conversely, the map $\mathbf{x}^{\mathrm{f}}=I_{\Omega_{0}^{\mathrm{f}}}+\mathbf{d}^{\mathrm{f}}$ can be defined from the interface displacement $\mathbf{d}_{\Gamma_{0}^{\mathrm{w}}}^{\mathrm{s}}$ as an arbitrary extension over the domain $\Omega_{0}^{\mathrm{f}}$, namely,

$$
\mathbf{d}^{\mathrm{f}}=\operatorname{Ext}\left(\mathbf{d}_{\mid \Gamma_{0}^{\mathrm{w}}}^{\mathrm{s}}\right) .
$$


This explains the use of the terminology Arbitrary Lagrangian Eulerian (ALE) formulation for the resulting equations $[8,20,22]$. In practice, we can choose an harmonic extension operator $[22,28,6,16]$, which means that $\mathbf{d}^{\mathrm{f}}$ solves the following elliptic problem:

$$
\left\{\begin{aligned}
-\kappa \Delta \mathbf{d}^{\mathrm{f}}=0, & \text { in } \quad \Omega_{0}^{\mathrm{f}}, \\
\mathbf{d}^{\mathrm{f}}=\mathbf{d}^{\mathrm{s}}, & \text { on } \quad \Gamma_{0}^{\mathrm{w}},
\end{aligned}\right.
$$

where $\kappa>0$ is a given "diffusion" coefficient, that might depend on $\mathbf{d}^{\mathrm{s}}$. Other alternative extension approaches can be found, for instance, in [2,34].

Therefore, the fluid domain can be parametrised as follows:

$$
\Omega^{\mathrm{f}}(t)=\Omega^{\mathrm{f}}\left(\mathbf{d}^{\mathrm{f}}(\cdot, t)\right)=\left(I_{\Omega_{0}^{\mathrm{f}}}+\mathbf{d}^{\mathrm{f}}\right)\left(\Omega_{0}^{\mathrm{f}}\right),
$$

and its corresponding velocity (the so-called ALE velocity) by

$$
\mathbf{w}\left(\mathbf{d}^{\mathbf{f}}\right) \stackrel{\text { def }}{=} \frac{\partial \mathbf{d}^{\mathrm{f}}}{\partial t} .
$$

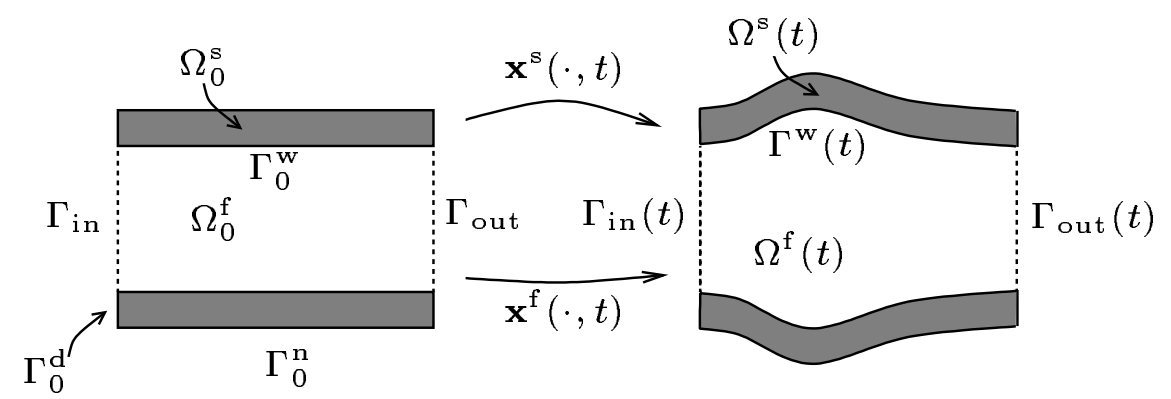

Figure 2: The map $\mathbf{x}$

We deal with a Newtonian viscous, homogeneous fluid under incompressible flow with density $\rho$ and kinetic viscosity $\mu$. Its behavior is described by its Eulerian velocity $\mathbf{u}$ and pressure $p$. The constitutive law for the Cauchy stress tensor is given by the following expression:

$$
\sigma(\mathbf{u}, p)=-p \mathrm{I}+\mu\left[\nabla \mathbf{u}+(\nabla \mathbf{u})^{\mathrm{T}}\right] .
$$

The elastic solid under large displacements is described by its displacement $\mathbf{d}^{\mathrm{s}}$ and its stress tensor $S$ (second Piola-Kirchhoff tensor). The field $S$ is related to $\mathbf{d}^{\mathrm{s}}$ through an appropriate constitutive law, $S=S\left(\mathbf{d}^{\mathrm{s}}\right)$ (see $[4,21,18]$ ). Its boundary is divided into three disjoint parts $\Gamma_{0}^{\mathrm{d}} \cup \Gamma_{0}^{\mathrm{n}} \cup \Gamma_{0}^{\mathrm{w}}$. We impose respectively a homogeneous Dirichlet boundary condition on $\Gamma_{0}^{\mathrm{d}}$ and a homogeneous Neumann boundary condition on $\Gamma_{0}^{\mathrm{n}}$. 
The coupling between the solid and the fluid is realized through standard boundary conditions at the fluid-structure interface $\Gamma_{0}^{\mathrm{w}}$, namely, the kinematic continuity of the velocity and the kinetic continuity of the stress:

$$
\begin{aligned}
\mathbf{u} & =\mathbf{w}\left(\mathbf{d}^{\mathrm{f}}\right), \\
F\left(\mathbf{d}^{\mathrm{s}}\right) S\left(\mathbf{d}^{\mathrm{s}}\right) \mathbf{n}_{0} & =J\left(\mathbf{d}^{\mathrm{f}}\right) \sigma(\mathbf{u}, p) F\left(\mathbf{d}^{\mathrm{f}}\right)^{-\mathrm{T}} \mathbf{n}_{0},
\end{aligned}
$$

with $\mathbf{n}_{0}$ being the unit outward normal vector to $\Omega_{0}^{\mathrm{f}}$, and

$$
F\left(\mathbf{d}^{\mathrm{s}}\right) \stackrel{\text { def }}{=} \mathrm{I}+\nabla_{0} \mathbf{d}^{\mathrm{s}}, \quad F\left(\mathbf{d}^{\mathrm{f}}\right) \stackrel{\text { def }}{=} \mathrm{I}+\nabla_{0} \mathbf{d}^{\mathrm{f}}, \quad J\left(\mathbf{d}^{\mathrm{s}}\right) \stackrel{\text { def }}{=} \operatorname{det} F\left(\mathbf{d}^{\mathrm{s}}\right), \quad J\left(\mathbf{d}^{\mathrm{f}}\right) \stackrel{\text { def }}{=} \operatorname{det} F\left(\mathbf{d}^{\mathrm{f}}\right),
$$

where $F\left(\mathbf{d}^{\mathrm{s}}\right)$ and $F\left(\mathbf{d}^{\mathrm{f}}\right)$ stand, respectively, for the deformation gradient inside the solid and the fluid domains.

Finally, the fluid-structure coupled state $\left(\mathbf{u}, p, \mathbf{d}^{\mathrm{f}} ; \mathbf{d}^{\mathrm{s}}\right)$ satisfies the following strong coupled system (involving an ALE fluid formulation),

$$
\left\{\begin{aligned}
\rho \frac{\partial J\left(\mathbf{d}^{\mathbf{f}}\right) \mathbf{u}}{\partial t}{ }_{\mid \mathbf{x}_{0}}+\rho \operatorname{div}\left[\mathbf{u} \otimes\left(\mathbf{u}-\mathbf{w}\left(\mathbf{d}^{\mathbf{f}}\right)\right)-\sigma(\mathbf{u}, p)\right]=\mathbf{0}, & \text { in } \quad \Omega^{\mathrm{f}}(t), \\
\operatorname{div} \mathbf{u}=0, & \text { in } \quad \Omega^{\mathrm{f}}(t), \\
\mathbf{u}=\mathbf{w}\left(\mathbf{d}^{\mathrm{f}}\right), & \text { on } \quad \Gamma_{0}^{\mathrm{w}}, \\
\sigma(\mathbf{u}, p) \mathbf{n}=\mathbf{g}, & \text { on } \quad \Gamma_{\text {in }}(t) \cup \Gamma_{\text {out }}(t), \\
\mathbf{d}^{\mathrm{f}}=\operatorname{Ext}\left(\mathbf{d}_{\left.\mid \Gamma_{0}^{\mathrm{s}}\right),}^{\mathrm{w}}\right) & \text { in } \quad \Omega_{0}^{\mathrm{f}}, \\
\rho_{0} \frac{\partial^{2} \mathbf{d}^{\mathrm{s}}}{\partial t^{2}}-\operatorname{div}_{0}\left(F\left(\mathbf{d}^{\mathrm{s}}\right) S\left(\mathbf{d}^{\mathrm{s}}\right)\right)=\mathbf{0}, & \text { in } \quad \Omega_{0}^{\mathrm{s}}, \\
F\left(\mathbf{d}^{\mathrm{s}}\right) S\left(\mathbf{d}^{\mathrm{s}}\right) \mathbf{n}_{0}=J\left(\mathbf{d}^{\mathrm{f}}\right) \sigma(\mathbf{u}, p) F\left(\mathbf{d}^{\mathrm{f}}\right)^{-\mathrm{T}} \mathbf{n}_{0}, & \text { on } \quad \Gamma_{0}^{\mathrm{w}}, \\
\mathbf{d}^{\mathrm{s}}=\mathbf{0}, & \text { on } \quad \Gamma_{0}^{\mathrm{d}}, \\
F\left(\mathbf{d}^{\mathrm{s}}\right) S\left(\mathbf{d}^{\mathrm{s}}\right) \mathbf{n}_{0}=\mathbf{0}, & \text { on } \quad \Gamma_{0}^{\mathrm{n}},
\end{aligned}\right.
$$

with $\rho_{0}$ the solid density, $\mathbf{n}$ the unit outward normal vector to $\Omega^{\mathrm{f}}(t)$, given by

$$
\mathbf{n}=\frac{F^{-\mathrm{T}}\left(\mathbf{d}^{\mathrm{f}}\right) \mathbf{n}_{0}}{\left\|F^{-\mathrm{T}}\left(\mathbf{d}^{\mathrm{f}}\right) \mathbf{n}_{0}\right\|}
$$

and $\mathbf{g}$ standing for the external forces acting on the fluid. For the sake of simpleness, no body forces are considered. In the sequel, we shall set $\Gamma_{\text {in-out }} \stackrel{\text { def }}{=} \Gamma_{\text {in }} \cup \Gamma_{\text {out }}$.

Remark 1 Let us notice that, in (3), the fluid domain $\Omega^{\mathrm{f}}(t)$ is directly related to the unknown $\mathbf{d}^{\mathrm{f}}$ through expression (1).

Remark 2 A variant to bypass the fluid ALE formulation in (3) is to use a fully Eulerian fluid description together with a space-time finite elements discretization, as described in [32, 33, 31]. 


\section{Continuous weak formulation}

Problem (3) can be reformulated in a weak variational form using appropriate test functions (see $[15,22]$ ), performing integration by parts and taking into account the boundary and interface conditions. This lead to the following coupled weak formulation (see $[28,22]$ ), involving a fluid and a solid weak-formulations: Find $\mathbf{d}^{\mathrm{f}}: \Omega_{0}^{\mathrm{f}} \longrightarrow \mathbb{R}^{3}, \mathbf{u}: \Omega^{\mathrm{f}}(t) \longrightarrow \mathbb{R}^{3}$, $p: \Omega^{\mathrm{f}}(t) \longrightarrow \mathbb{R}$ and $\mathbf{d}^{\mathrm{s}}: \Omega_{0}^{\mathrm{s}} \longrightarrow \mathbb{R}^{3}$, satisfying the following coupled non-linear subproblems:

1. The fluid weak-formulation:

$$
\begin{aligned}
& \rho \frac{\mathrm{d}}{\mathrm{d} t} \int_{\Omega^{\mathrm{f}}(t)} \mathbf{u} \cdot \mathbf{v}^{\mathrm{f}} \mathrm{d} \mathbf{x}+\rho \int_{\Omega^{\mathrm{f}}(t)} \operatorname{div}\left[\mathbf{u} \otimes\left(\mathbf{u}-\mathbf{w}\left(\mathbf{d}^{\mathrm{f}}\right)\right)\right] \cdot \mathbf{v}^{\mathrm{f}} \mathrm{d} \mathbf{x} \\
& +\int_{\Omega^{\mathrm{f}}(t)} \sigma(\mathbf{u}, p): \nabla \mathbf{v}^{\mathrm{f}} \mathrm{d} \mathbf{x}-\int_{\Gamma_{\text {in }- \text { out }}(t)} \mathbf{g}(t) \cdot \mathbf{v}^{\mathrm{f}} \mathrm{d} \mathbf{a}-\int_{\Omega^{\mathrm{f}}(t)} q \operatorname{div} \mathbf{u} \mathrm{d} \mathbf{x} \\
& +\int_{\Gamma^{\mathrm{w}}(t)}\left(\mathbf{u}-\mathbf{w}\left(\mathbf{d}^{\mathrm{f}}\right)\right) \cdot \boldsymbol{\xi} \mathrm{d} \mathbf{a}+\int_{\Omega_{0}^{\mathrm{f}}}\left(\mathbf{d}^{\mathrm{f}}-\operatorname{Ext}\left(\mathbf{d}_{\mid \Gamma_{0}^{\mathrm{w}}}^{\mathrm{s}}\right)\right) \cdot \boldsymbol{\lambda} \mathrm{d} \mathbf{x}_{0}=0, \\
& \forall\left(\mathbf{v}^{\mathrm{f}}, q, \boldsymbol{\xi}, \boldsymbol{\lambda}\right) \in V^{\mathrm{f}}(t) .
\end{aligned}
$$

2. The solid weak-formulation:

$$
\begin{aligned}
& \int_{\Omega_{0}^{\mathrm{s}}} \rho_{0} \frac{\partial^{2} \mathbf{d}^{\mathrm{s}}}{\partial t^{2}} \cdot \mathbf{v}^{\mathrm{s}} \mathrm{d} \mathbf{x}_{0}+\int_{\Omega_{0}^{\mathrm{s}}} F\left(\mathbf{d}^{\mathrm{s}}\right) S\left(\mathbf{d}^{\mathrm{s}}\right): \nabla \mathbf{v}^{\mathrm{s}} \mathrm{d} \mathbf{x}_{0} \\
& +\rho \frac{\mathrm{d}}{\mathrm{d} t} \int_{\Omega^{\mathrm{f}}(t)} \mathbf{u} \cdot \mathscr{R}\left(\mathbf{v}_{\mid \Gamma_{0}^{\mathrm{w}}}^{\mathrm{s}}\right) \mathrm{d} \mathbf{x}+\rho \int_{\Omega^{\mathrm{f}}(t)} \operatorname{div}\left[\mathbf{u} \otimes\left(\mathbf{u}-\mathbf{w}\left(\mathbf{d}^{\mathrm{f}}\right)\right)\right] \cdot \mathscr{R}\left(\mathbf{v}_{\mid \Gamma_{0}^{\mathrm{w}}}^{\mathrm{s}}\right) \mathrm{d} \mathbf{x} \\
& +\int_{\Omega^{\mathrm{f}}(t)} \sigma(\mathbf{u}, p): \nabla \mathscr{R}\left(\mathbf{v}_{\mid \Gamma_{0}^{\mathrm{w}}}^{\mathrm{s}}\right) \mathrm{d} \mathbf{x}=0, \quad \forall \mathbf{v}^{\mathrm{s}} \in V^{\mathrm{s}} .
\end{aligned}
$$

Here, the space of solid test functions is given by

$$
V^{\mathrm{s}} \stackrel{\text { def }}{=}\left\{\mathbf{v}^{\mathrm{s}} \in H^{1}\left(\Omega_{0}^{\mathrm{s}}\right)^{3} \mid \mathbf{v}^{\mathrm{s}}=0 \text { on } \Gamma_{0}^{\mathrm{d}}\right\},
$$

and the space of fluid test functions $V^{\mathrm{f}}(t)$ is defined using the ALE parametrization $\mathbf{x}^{\mathrm{f}}$ (see [15]),

$$
\begin{aligned}
V^{\mathrm{f}}(t) \stackrel{\text { def }}{=} & \left\{\mathbf{v}^{\mathrm{f}}=\hat{\mathbf{v}} \circ\left(\mathbf{x}^{\mathrm{f}}\right)^{-1} \mid \hat{\mathbf{v}} \in H^{1}\left(\Omega_{0}^{\mathrm{f}}\right)^{3}, \hat{\mathbf{v}}=0 \text { on } \Gamma_{0}^{\mathrm{w}}\right\} \\
& \times\left\{q=\hat{q} \circ\left(\mathbf{x}^{\mathrm{f}}\right)^{-1} \mid \hat{q} \in L^{2}\left(\Omega_{0}^{\mathrm{f}}\right)\right\} \\
& \times\left\{\boldsymbol{\xi}=\hat{\boldsymbol{\xi}} \circ\left(\mathbf{x}^{\mathrm{f}}\right)_{\mid \Gamma^{\mathrm{w}}(t)}^{-1} \mid \hat{\boldsymbol{\xi}} \in L^{2}\left(\Gamma_{0}^{\mathrm{w}}\right)^{3}\right\} \\
& \times L^{2}\left(\Omega_{0}^{\mathrm{f}}\right)^{3} .
\end{aligned}
$$

In addition, we denote by $\mathscr{R}$ a given linear continuous lift operator

$$
\mathscr{R}: H^{\frac{1}{2}}\left(\Gamma_{0}^{\mathrm{W}}\right) \longrightarrow\left\{\mathbf{v}^{\mathrm{f}}=\hat{\mathbf{v}} \circ\left(\mathbf{x}^{\mathrm{f}}\right)^{-1} \mid \hat{\mathbf{v}} \in H^{1}\left(\Omega_{0}^{\mathrm{f}}\right)^{3}, \hat{\mathbf{v}}=0 \text { on } \Gamma_{\text {in }- \text { out }}\right\} .
$$


Remark 3 Notice that the interface coupling condition $(2)_{2}$ is implicitly treated in (5). Indeed, the last three terms of equation (5) represent nothing but the residual of the fluid momentum equations in (4). This furnishes a weak description of the fluid load at the interface (see [22]).

\section{Time semi-discretised weak formulation}

The weak coupled formulation (4)-(5) is now semi-discretized in time. Numerical experiments (see $[28,22,23,24,16,6]$ ) show that only fully coupled schemes (e.g. the fluid geometry and the interface coupling conditions are implicitly treated) ensure stability and allow to solve effectively problems in which both the fluid and structural densities are of the same order. This arises, for instance, in haemodynamics applications (see $[28,14,6,16,17])$.

\subsection{An implicit coupling scheme}

We use an implicit Euler scheme for the ALE Navier-Stokes equations, with a semi-implicit treatment of the non-linear convective term. Furthermore we use a mid-point rule for the structural equation (see $[22,14,16,6])$. Thus, for $n=0,1, \ldots$, the semi-discretized in time problem writes: Given $\left(\mathbf{u}^{n}, \mathbf{d}^{\mathrm{f}, n} ; \mathbf{d}^{\mathrm{s}, n}, \mathbf{w}^{\mathrm{s}, n}\right)$, find

$$
\left(\mathbf{u}^{n+1}, p^{n+1}, \mathbf{d}^{\mathrm{f}, n+1} ; \mathbf{d}^{\mathrm{s}, n+1}\right),
$$

satisfying the following coupled non-linear subproblems:

1. Fluid time semi-discretised weak-formulation:

$$
\begin{aligned}
& \frac{\rho}{\Delta t} \int_{\Omega^{\mathrm{f}}\left(t_{n+1}\right)} \mathbf{u}^{n+1} \cdot \mathbf{v}^{\mathrm{f}} \mathrm{d} \mathbf{x}-\frac{\rho}{\Delta t} \int_{\Omega^{\mathrm{f}}\left(t_{n}\right)} \mathbf{u}^{n} \cdot \mathbf{v}^{\mathrm{f}} \mathrm{d} \mathbf{x} \\
& +\rho \int_{\Omega^{\mathrm{f}}\left(t_{n+1}\right)} \operatorname{div}\left[\mathbf{u}^{n+1} \otimes\left(\mathbf{u}^{n}-\mathbf{w}_{\mathrm{g}}\left(\mathbf{d}^{\mathrm{f}, n+1}\right)\right)\right] \cdot \mathbf{v}^{\mathrm{f}} \mathrm{d} \mathbf{x} \\
& +\int_{\Omega^{\mathrm{f}}\left(t_{n+1}\right)} \sigma\left(\mathbf{u}^{n+1}, p^{n+1}\right): \nabla \mathbf{v}^{\mathrm{f}} \mathrm{d} \mathbf{x}-\int_{\Gamma_{\text {in }- \text { out }}\left(t_{n+1}\right)} \mathbf{g}\left(t_{n+1}\right) \cdot \mathbf{v}^{\mathrm{f}} \mathrm{d} \mathbf{a} \\
& -\int_{\Omega^{\mathrm{f}}\left(t_{n+1}\right)} q \operatorname{div} \mathbf{u}^{n+1} \mathrm{~d} \mathbf{x}+\int_{\Gamma^{\mathrm{w}}\left(t_{n+1}\right)}\left(\mathbf{u}^{n+1}-\mathbf{w}_{\mathrm{g}}\left(\mathbf{d}^{\mathrm{f}, n+1}\right)\right) \cdot \boldsymbol{\xi} \mathrm{d} \mathbf{a} \\
& +\int_{\Omega_{0}^{\mathrm{f}}}\left(\mathbf{d}^{\mathrm{f}, n+1}-\operatorname{Ext}\left(\mathbf{d}_{\mid \Gamma_{0}^{\mathrm{w}}}^{\mathrm{s}, n+1}\right)\right) \cdot \boldsymbol{\lambda} \mathrm{d} \mathbf{x}_{0}=0, \quad \forall\left(\mathbf{v}^{\mathrm{f}}, q, \boldsymbol{\xi}, \boldsymbol{\lambda}\right) \in V^{\mathrm{f}}\left(t_{n+1}\right),
\end{aligned}
$$

with the notation $\mathbf{w}_{\mathrm{g}}\left(\mathbf{d}^{\mathrm{f}, n+1}\right) \stackrel{\text { def }}{=} \frac{1}{\Delta t}\left(\mathbf{d}^{\mathrm{f}, n+1}-\mathbf{d}^{\mathrm{f}, n}\right)$. 
2. Solid time semi-discretised weak-formulation:

$$
\begin{aligned}
& \frac{2}{(\Delta t)^{2}} \int_{\Omega_{0}^{\mathrm{s}}} \rho_{0} \mathbf{d}^{\mathrm{s}, n+1} \cdot \mathbf{v}^{\mathrm{s}} \mathrm{d} \mathbf{x}_{0}-\frac{2}{(\Delta t)^{2}} \int_{\Omega_{0}^{\mathrm{s}}} \rho_{0}\left(\mathbf{d}^{\mathrm{s}, n}+\Delta t \mathbf{w}^{\mathrm{s}, n}\right) \cdot \mathbf{v}^{\mathrm{s}} \mathrm{d} \mathbf{x}_{0} \\
& +\frac{1}{2} \int_{\Omega_{0}^{\mathrm{s}}} F\left(\mathbf{d}^{\mathrm{s}, n+1}\right) S\left(\mathbf{d}^{\mathrm{s}, n+1}\right): \nabla \mathbf{v}^{\mathrm{s}} \mathrm{d} \mathbf{x}_{0}+\frac{1}{2} \int_{\Omega_{0}^{\mathrm{s}}} F\left(\mathbf{d}^{\mathrm{s}, n}\right) S\left(\mathbf{d}^{\mathrm{s}, n}\right): \nabla \mathbf{v}^{\mathrm{s}} \mathrm{d} \mathbf{x}_{0} \\
& +\frac{\rho}{\Delta t} \int_{\Omega^{\mathrm{f}}\left(t_{n}+1\right)} \mathbf{u}^{n+1} \cdot \mathscr{R}\left(\mathbf{v}_{\mid \Gamma_{0}^{\mathrm{w}}}^{\mathrm{s}}\right) \mathrm{d} \mathbf{x}-\frac{\rho}{\Delta t} \int_{\Omega^{\mathrm{f}}\left(t_{n}\right)} \mathbf{u}^{n} \cdot \mathscr{R}\left(\mathbf{v}_{\mid \Gamma_{0}^{\mathrm{w}}}^{\mathrm{s}}\right) \mathrm{d} \mathbf{x} \\
& +\rho \int_{\Omega^{\mathrm{f}}\left(t_{n+1}\right)} \operatorname{div}\left[\mathbf{u}^{n+1} \otimes\left(\mathbf{u}^{n}-\mathbf{w}_{\mathrm{g}}\left(\mathbf{d}^{\mathrm{f}, n+1}\right)\right)\right] \cdot \mathscr{R}\left(\mathbf{v}_{\mid \Gamma_{0}^{\mathrm{w}}}^{\mathrm{s}}\right) \mathrm{d} \mathbf{x} \\
& +\int_{\Omega^{\mathrm{f}}\left(t_{n+1}\right)} \sigma\left(\mathbf{u}^{n+1}, p^{n+1}\right): \nabla \mathscr{R}\left(\mathbf{v}_{\mid \Gamma_{0}^{\mathrm{w}}}^{\mathrm{s}}\right) \mathrm{d} \mathbf{x}=0, \quad \forall \mathbf{v}^{\mathrm{s}} \in V^{\mathrm{s}},
\end{aligned}
$$

with $\mathbf{w}^{\mathrm{s}, n+1}=\frac{2}{\Delta t}\left(\mathbf{d}^{\mathrm{s}, n+1}-\mathbf{d}^{\mathrm{s}, n}\right)-\mathbf{w}^{\mathrm{s}, n}$.

\subsection{Abstract formulation}

In order to use general methods to solve the coupled-system (6)-(7), it is useful to turn it into an abstract form. Let $U^{\mathrm{f}} \times U^{\mathrm{s}}$ be the space of fluid and solid states. We introduce the following operators:

1. Fluid operator:

$$
\begin{aligned}
\mathcal{F}: U^{\mathrm{f}} \times U^{\mathrm{s}} & \longrightarrow\left(V^{\mathrm{f}}\right)^{\prime} \\
\left(u^{\mathrm{f}}, u^{\mathrm{s}}\right) & \longmapsto \mathcal{F}\left(u^{\mathrm{f}}, u^{\mathrm{s}}\right),
\end{aligned}
$$

with $u^{\mathrm{f}} \stackrel{\text { def }}{=}\left(\mathbf{u}, p, \mathbf{d}^{\mathrm{f}}\right)$ and $u^{\mathrm{s}} \stackrel{\text { def }}{=} \mathbf{d}^{\mathrm{s}}$, whose action on fluid test functions (in $\left.V^{\mathrm{f}}\left(t_{n+1}\right)\right)$ is defined as follows,

$$
\begin{aligned}
& \left\langle\mathcal{F}\left(\mathbf{u}, p, \mathbf{d}^{\mathrm{f}} ; \mathbf{d}^{\mathrm{s}}\right),\left(\mathbf{v}^{\mathrm{f}}, q, \boldsymbol{\xi}, \boldsymbol{\lambda}\right)\right\rangle \stackrel{\text { def }}{=} \frac{\rho}{\Delta t} \int_{\Omega^{\mathrm{f}}\left(\mathbf{d}^{\mathrm{f}}\right)} \mathbf{u} \cdot \mathbf{v}^{\mathrm{f}} \mathrm{d} \mathbf{x}-\frac{\rho}{\Delta t} \int_{\Omega^{\mathrm{f}}\left(t_{n}\right)} \mathbf{u}^{n} \cdot \mathbf{v}^{\mathrm{f}} \mathrm{d} \mathbf{x} \\
& +\rho \int_{\Omega^{\mathrm{f}}\left(\mathbf{d}^{\mathrm{f}}\right)} \operatorname{div}\left[\mathbf{u} \otimes\left(\mathbf{u}^{n}-\mathbf{w}_{\mathrm{g}}\left(\mathbf{d}^{\mathrm{f}}\right)\right)\right] \cdot \mathbf{v}^{\mathrm{f}} \mathrm{d} \mathbf{x}+\int_{\Omega^{\mathrm{f}}\left(\mathbf{d}^{\mathrm{f}}\right)} \sigma(\mathbf{u}, p): \nabla \mathbf{v}^{\mathrm{f}} \mathrm{d} \mathbf{x} \\
& -\int_{\Gamma_{\text {in }- \text { out }}\left(\mathbf{d}^{\mathrm{f}}\right)} \mathbf{g}\left(t_{n+1}\right) \cdot \mathbf{v}^{\mathrm{f}} \mathrm{d} \mathbf{a}-\int_{\Omega^{\mathrm{f}}\left(\mathbf{d}^{\mathrm{f}}\right)} q \operatorname{div} \mathbf{u} \mathrm{d} \mathbf{x} \\
& +\int_{\Gamma^{\mathrm{w}}\left(\mathbf{d}^{\mathrm{f}}\right)}\left(\mathbf{u}-\mathbf{w}_{\mathrm{g}}\left(\mathbf{d}^{\mathrm{f}}\right)\right) \cdot \boldsymbol{\xi} \mathrm{d} \mathbf{a}+\int_{\Omega_{0}^{\mathrm{f}}}\left(\mathbf{d}^{\mathrm{f}}-\operatorname{Ext}\left(\mathbf{d}_{\mid \Gamma_{0}^{\mathrm{w}}}^{\mathrm{s}}\right)\right) \cdot \boldsymbol{\lambda} \mathrm{d} \mathbf{x}_{0},
\end{aligned}
$$

with $\mathbf{w}_{\mathrm{g}}\left(\mathbf{d}^{\mathrm{f}}\right)=\frac{1}{\Delta t}\left(\mathbf{d}^{\mathrm{f}}-\mathbf{d}^{\mathrm{f}, n}\right)$.

2. Solid operator:

$$
\begin{aligned}
\mathcal{S}: \begin{array}{c}
U^{\mathrm{f}} \times U^{\mathrm{s}} \\
\left(u^{\mathrm{f}}, u^{\mathrm{s}}\right)
\end{array} & \longmapsto \mathcal{S}\left(V^{\mathrm{s}}\right)^{\prime} \\
& \longmapsto \mathcal{S}\left(u^{\mathrm{f}}, u^{\mathrm{s}}\right),
\end{aligned}
$$


whose action on solid test functions is defined as follows,

$$
\begin{aligned}
& \left\langle\mathcal{S}\left(\mathbf{u}, p, \mathbf{d}^{\mathrm{f}} ; \mathbf{d}^{\mathrm{s}}\right), \mathbf{v}^{\mathrm{s}}\right\rangle \stackrel{\text { def }}{=} \frac{2}{(\Delta t)^{2}} \int_{\Omega_{0}^{\mathrm{s}}} \rho_{0} \mathbf{d}^{\mathrm{s}} \cdot \mathbf{v}^{\mathrm{s}} \mathrm{d} \mathbf{x}_{0} \\
& -\frac{2}{(\Delta t)^{2}} \int_{\Omega_{0}^{\mathrm{s}}} \rho_{0}\left(\mathbf{d}^{\mathrm{s}, n}+\Delta t \mathbf{w}^{\mathrm{s}, n}\right) \cdot \mathbf{v}^{\mathrm{s}} \mathrm{d} \mathbf{x}_{0}+\frac{1}{2} \int_{\Omega_{0}^{\mathrm{s}}} F\left(\mathbf{d}^{\mathrm{s}}\right) S\left(\mathbf{d}^{\mathrm{s}}\right): \nabla \mathbf{v}^{\mathrm{s}} \mathrm{d} \mathbf{x}_{0} \\
& +\frac{1}{2} \int_{\Omega_{0}^{\mathrm{s}}} F\left(\mathbf{d}^{\mathrm{s}, n}\right) S\left(\mathbf{d}^{\mathrm{s}, n}\right): \nabla \mathbf{v}^{\mathrm{s}} \mathrm{d} \mathbf{x}_{0}+\left\langle\mathcal{L}\left(\mathbf{u}, p, \mathbf{d}^{\mathrm{f}}\right), \mathbf{v}^{\mathrm{s}}\right\rangle,
\end{aligned}
$$

where the fluid load transfer operator $\mathcal{L}: U^{\mathrm{f}} \longrightarrow\left(V^{\mathrm{s}}\right)^{\prime}$ is defined by

$$
\begin{aligned}
& \left\langle\mathcal{L}\left(\mathbf{u}, p, \mathbf{d}^{\mathrm{f}}\right), \mathbf{v}^{\mathrm{s}}\right\rangle \stackrel{\text { def }}{=}\left\langle\mathcal{F}\left(\mathbf{u}, p, \mathbf{d}^{\mathrm{f}} ; \mathbf{d}^{\mathrm{s}}\right),\left(\mathscr{R}\left(\mathbf{v}_{\mid \Gamma_{0}^{\mathrm{w}}}^{\mathrm{s}}\right), 0, \mathbf{0}, \mathbf{0}\right)\right\rangle \\
& =\frac{\rho}{\Delta t} \int_{\Omega^{\mathrm{f}}\left(\mathbf{d}^{\mathrm{f}}\right)} \mathbf{u} \cdot \mathscr{R}\left(\mathbf{v}_{\mid \Gamma_{0}^{\mathrm{w}}}^{\mathrm{s}}\right) \mathrm{d} \mathbf{x}-\frac{\rho}{\Delta t} \int_{\Omega^{\mathrm{f}}\left(t_{n}\right)} \mathbf{u}^{n} \cdot \mathscr{R}\left(\mathbf{v}_{\mid \Gamma_{0}^{\mathrm{w}}}^{\mathrm{s}}\right) \mathrm{d} \mathbf{x} \\
& +\rho \int_{\Omega^{\mathrm{f}}\left(\mathbf{d}^{\mathrm{f}}\right)} \operatorname{div}\left[\mathbf{u} \otimes\left(\mathbf{u}^{n}-\mathbf{w}_{\mathrm{g}}\left(\mathbf{d}^{\mathrm{f}}\right)\right)\right] \cdot \mathscr{R}\left(\mathbf{v}_{\mid \Gamma_{0}^{\mathrm{w}}}^{\mathrm{s}}\right) \mathrm{d} \mathbf{x} \\
& +\int_{\Omega^{\mathrm{f}}\left(\mathbf{d}^{\mathrm{f}}\right)} \sigma(\mathbf{u}, p): \nabla \mathscr{R}\left(\mathbf{v}_{\mid \Gamma_{0}^{\mathrm{s}}}^{\mathrm{w}}\right) \mathrm{d} \mathbf{x} .
\end{aligned}
$$

Therefore, using the above definitions, the coupled problem (6)-(7) reads: For $n=0,1, \ldots$, find

$$
\left(\mathbf{u}^{n+1}, p^{n+1}, \mathbf{d}^{\mathrm{f}, n+1} ; \mathbf{d}^{\mathrm{s}, n+1}\right),
$$

solution of the following non-linear coupled system:

$$
\begin{array}{rlrl}
\left\langle\mathcal{F}\left(\mathbf{u}^{n+1}, p^{n+1}, \mathbf{d}^{\mathrm{f}, n+1} ; \mathbf{d}^{\mathrm{s}, n+1}\right),\left(\mathbf{v}^{\mathrm{f}}, q, \boldsymbol{\xi}, \boldsymbol{\lambda}\right)\right\rangle & =0, & & \forall\left(\mathbf{v}^{\mathrm{f}}, q, \boldsymbol{\xi}, \boldsymbol{\lambda}\right) \in V^{\mathrm{f}}\left(t_{n+1}\right), \\
\left\langle\mathcal{S}\left(\mathbf{u}^{n+1}, p^{n+1}, \mathbf{d}^{\mathrm{f}, n+1} ; \mathbf{d}^{\mathrm{s}, n+1}\right), \mathbf{v}^{\mathrm{s}}\right\rangle=0, & \forall \mathbf{v}^{\mathrm{s}} \in V^{\mathrm{s}},
\end{array}
$$

that is,

$$
\begin{aligned}
& \mathcal{F}\left(\mathbf{u}^{n+1}, p^{n+1}, \mathbf{d}^{\mathrm{f}, n+1} ; \mathbf{d}^{\mathrm{s}, n+1}\right)=0 \\
& \mathcal{S}\left(\mathbf{u}^{n+1}, p^{n+1}, \mathbf{d}^{\mathrm{f}, n+1} ; \mathbf{d}^{\mathrm{s}, n+1}\right)=0 .
\end{aligned}
$$

To simplify the notation, we shall only consider one time step so that we drop the upper index $n+1$. Thus, the above coupled problem writes: find $u^{\mathrm{f}} \stackrel{\text { def }}{=}\left(\mathbf{u}, p, \mathbf{d}^{\mathrm{f}}\right)$ and $u^{\mathrm{s}} \stackrel{\text { def }}{=} \mathbf{d}^{\mathrm{s}}$ such that

$$
\begin{gathered}
\mathcal{F}\left(u^{\mathrm{f}}, u^{\mathrm{s}}\right)=0, \\
\mathcal{S}\left(u^{\mathrm{f}}, u^{\mathrm{s}}\right)=0 .
\end{gathered}
$$

In the sequel, we shall assume that a fluid solver and a solid solver are at our disposal. Hence, it is useful to turn problem (11) into an abstract form in terms of the fluid and 
structure solvers. To this end, we introduce the fluid solver operator $\mathscr{F}: U^{\mathrm{s}} \longrightarrow U^{\mathrm{f}}$, defined by

$$
\mathcal{F}\left(\mathscr{F}\left(u^{\mathrm{s}}\right), u^{\mathrm{s}}\right)=0, \quad \forall u^{\mathrm{s}} \in U^{\mathrm{s}},
$$

and the solid solver operator $\mathscr{S}: U^{\mathrm{f}} \longrightarrow U^{\mathrm{s}}$, defined by

$$
\mathcal{S}\left(u^{\mathrm{f}}, \mathscr{S}\left(u^{\mathrm{f}}\right)\right)=0, \quad \forall u^{\mathrm{f}} \in U^{\mathrm{f}} .
$$

Therefore, using the above definitions, the coupled non-linear problem (11) can be reduced to: find $u^{\mathrm{s}} \in U^{\mathrm{s}}$ solution of the following non-linear equation

$$
\mathcal{R}\left(u^{\mathrm{s}}\right) \stackrel{\text { def }}{=} u^{\mathrm{s}}-\mathscr{S} \circ \mathscr{F}\left(u^{\mathrm{s}}\right)=0,
$$

see [16]. If $\left(u^{\mathrm{f}}, u^{\mathrm{s}}\right)$ solves (11) then $u^{\mathrm{s}}$ satisfies (14) and, conversely, if $u^{\mathrm{s}}$ is a solution of (14) then $\left(u^{\mathrm{f}}=\mathscr{F}\left(u^{\mathrm{s}}\right), u^{\mathrm{s}}\right)$ solves $(11)$.

In the next section, we describe the main steps of the Newton's algorithm when applied to the above nonlinear problem.

\section{The Newton's algorithm}

Standard forms for solving the non-linear problem (14) are fixed-points based iterations, see $[28,25,16,6]$. Unfortunately, these methods usually show poor convergence properties and may fail to converge (see $[23,24,25,6,16]$ ). In order to speed up the convergence, it is useful to use Newton-Raphson based methods (see [23, 24, 31, 16, 19]).

The Newton's method applied to the non-linear equation (14) reads:

1. Choose $\bar{u}^{\mathrm{s}} \in U^{\mathrm{s}}$

2. Do until convergence
(a) Evaluate the fluid operator $\bar{u}^{\mathrm{f}}=\mathscr{F}\left(\bar{u}^{\mathrm{s}}\right)$
(b) Evaluate the solid operator $\hat{u}^{\mathrm{s}}=\mathscr{S}\left(\bar{u}^{\mathrm{f}}\right)$
(c) Evaluate the residual $\mathcal{R}\left(\bar{u}^{\mathrm{s}}\right)=\bar{u}^{\mathrm{s}}-\hat{u}^{\mathrm{s}}$
(d) Solve

$$
\left[\mathrm{D}_{u^{\mathrm{s}}} \mathcal{R}\left(\bar{u}^{\mathrm{s}}\right)\right] \delta u^{\mathrm{s}}=-\mathcal{R}\left(\bar{u}^{\mathrm{s}}\right)
$$
(c) Update rule: $\bar{u}^{\mathrm{s}} \longleftarrow \bar{u}^{\mathrm{s}}+\delta u^{\mathrm{s}}$.

Step 2(b) of this algorithm can be carried out by using an iterative free matrix method as GMRES (see [23, 24, 31, 16]). In this case, we only need to evaluate several times the operator $\mathrm{D}_{u^{s}} \mathcal{R}\left(\bar{u}^{\mathrm{s}}\right)$ against solid state perturbations $z$. In particular, we have,

$$
\left[\mathrm{D}_{u^{\mathrm{s}}} \mathcal{R}\left(\bar{u}^{\mathrm{s}}\right)\right] z=z-\underbrace{\left[\mathrm{D}_{u^{\mathrm{f}}} \mathscr{S}\left(\bar{u}^{\mathrm{f}}\right)\right] \underbrace{\left[\mathrm{D}_{u^{\mathrm{s}}} \mathscr{F}\left(\bar{u}^{\mathrm{s}}\right)\right] z}_{\delta u^{\mathrm{f}}=\left(\delta \mathbf{u}, \delta p, \delta \mathbf{d}^{\mathrm{f}}\right)}}_{\delta z} .
$$

$\mathrm{RR} \mathrm{n}^{\circ} 5087$ 
By differentiating the equations (12) and (13), we are able to split the above expression in terms of the derivatives of the fluid an solid operators (8)-(9), defined in the previous section:

(i) Solve the fluid tangent sub-problem:

$$
\left[\mathrm{D}_{u^{\mathrm{f}}} \mathcal{F}\left(\bar{u}^{\mathrm{f}}, \bar{u}^{\mathrm{s}}\right)\right] \delta u^{\mathrm{f}}=-\left[\mathrm{D}_{u^{\mathrm{s}}} \mathcal{F}\left(\bar{u}^{\mathrm{f}}, \bar{u}^{\mathrm{s}}\right)\right] z,
$$

(ii) Solve the solid tangent sub-problem:

$$
\left[\mathrm{D}_{u^{\mathrm{s}}} \mathcal{S}\left(\bar{u}^{\mathrm{f}}, \hat{u}^{\mathrm{s}}\right)\right] \delta z=-\left[\mathrm{D}_{u^{\mathrm{f}}} \mathcal{S}\left(\bar{u}^{\mathrm{f}}, \hat{u}^{\mathrm{s}}\right)\right] \delta u^{\mathrm{f}},
$$

(iii) Evaluate

$$
\left[\mathrm{D}_{u^{\mathrm{s}}} \mathcal{R}\left(\bar{u}^{\mathrm{s}}\right)\right] z=z-\delta z .
$$

The main difficulty here (see $[23,24,31,16,19])$ relies on the fluid tangent subproblem (15). Indeed, as we shall see in the next section, this problem involves the evaluation of the following cross-derivative of the fluid operator:

$$
\mathrm{D}_{\mathbf{d}^{\mathrm{f}}} \mathcal{F}\left(\overline{\mathbf{u}}, \bar{p}, \overline{\mathbf{d}^{\mathrm{f}}} ; \overline{\mathbf{d}^{\mathrm{s}}}\right) \delta \mathbf{d}^{\mathrm{f}},
$$

which corresponds to the directional derivative with respect to fluid-domain perturbations. In previous works, the evaluations of these cross-jacobians were performed using finite difference approximations, that only require state operators evaluations $[23,24,31,19]$. However, the lack of a priori rules for selecting optimal finite difference infinitesimal steps, leads to non-consistent jacobians and a reduction of the overall convergence speed [16]. In the following section, we will show how to avoid these approximations by establishing the exact expression of the above linearized sub-systems.

\section{Weak state operators derivatives}

In this section, we present the differentiation of the fluid and solid operators with respect to the fluid and solid state variables. We will use shape derivative calculus results in order to perform the differentiation of integral terms with respect to their supports.

\subsection{Fluid operator derivatives}

We are able to obtain the expressions of the action of the derivative of the fluid weak state operator $\mathcal{F}$ with respect to the state variables $u^{\mathrm{f}}=\left(\mathbf{u}, p, \mathbf{d}^{\mathrm{f}}\right)$ and $u^{\mathrm{s}} \stackrel{\text { def }}{=} \mathbf{d}^{\mathrm{s}}$ at point $\left(\overline{\mathbf{u}}, \bar{p}, \overline{\mathbf{d}}^{\mathrm{f}} ; \overline{\mathbf{d}}^{\mathrm{s}}\right)$ in the direction $\left(\delta \mathbf{u}, \delta p, \delta \mathbf{d}^{\mathrm{f}} ; \delta \mathbf{d}^{\mathrm{s}}\right)$. We can split the operator derivative in two terms:

$$
\begin{aligned}
\mathrm{D}_{\left(\mathbf{u}, p, \mathbf{d}^{\mathrm{f}}\right)} \mathcal{F}\left(\overline{\mathbf{u}}, \bar{p}, \overline{\mathbf{d}}^{\mathrm{f}} ; \overline{\mathbf{d}}^{\mathrm{s}}\right)\left(\delta \mathbf{u}, \delta p, \delta \mathbf{d}^{\mathrm{f}}\right)= & \mathrm{D}_{(\mathbf{u}, p)} \mathcal{F}\left(\overline{\mathbf{u}}, \bar{p}, \overline{\mathbf{d}}^{\mathrm{f}} ; \overline{\mathbf{d}}^{\mathrm{s}}\right)(\delta \mathbf{u}, \delta p) \\
& +\mathrm{D}_{\mathbf{d}^{\mathrm{f}}} \mathcal{F}\left(\overline{\mathbf{u}}, \bar{p}, \overline{\mathbf{d}}^{\mathrm{f}} ; \overline{\mathbf{d}}^{\mathrm{s}}\right) \delta \mathbf{d}^{\mathrm{f}}
\end{aligned}
$$


The first term $\mathrm{D}_{(\mathbf{u}, p)} \mathcal{F}\left(\overline{\mathbf{u}}, \bar{p}, \overline{\mathbf{d}^{\mathrm{f}}} ; \overline{\mathbf{d}^{\mathrm{s}}}\right)(\delta \mathbf{u}, \delta p)$ is a classical Fréchet derivative, in the sense we do not need to perturb the support of the integrals inside the operator. Thus, we have

$$
\begin{aligned}
& \left\langle\mathrm{D}_{(\mathbf{u}, p)} \mathcal{F}\left(\overline{\mathbf{u}}, \bar{p}, \overline{\mathbf{d}^{\mathrm{f}}} ; \overline{\mathbf{d}^{\mathrm{s}}}\right)(\delta \mathbf{u}, \delta p),\left(\mathbf{v}^{\mathrm{f}}, q, \boldsymbol{\xi}, \boldsymbol{\lambda}\right)\right\rangle=\frac{\rho}{\Delta t} \int_{\Omega^{\mathrm{f}}\left(\overline{\mathbf{d}}^{\mathrm{f}}\right)} \delta \mathbf{u} \cdot \mathbf{v}^{\mathrm{f}} \mathrm{d} \mathbf{x} \\
& +\rho \int_{\Omega^{\mathrm{f}}\left(\overline{\mathbf{d}}^{\mathrm{f}}\right)} \operatorname{div}\left[\delta \mathbf{u} \otimes\left(\mathbf{u}^{n}-\mathbf{w}_{\mathrm{g}}\left(\overline{\mathbf{d}^{\mathrm{f}}}\right)\right)\right] \cdot \mathbf{v}^{\mathrm{f}} \mathrm{d} \mathbf{x}+\int_{\Omega^{\mathrm{f}}\left(\overline{\mathbf{d}}^{\mathrm{f}}\right)} \sigma(\delta \mathbf{u}, \delta p): \nabla \mathbf{v}^{\mathrm{f}} \mathrm{d} \mathbf{x} \\
& -\int_{\Omega^{\mathrm{f}}\left(\overline{\mathbf{d}}^{\mathrm{f}}\right)} q \operatorname{div} \delta \mathbf{u} \mathrm{d} \mathbf{x}+\int_{\Gamma^{\mathrm{w}}\left(\overline{\mathbf{d}}^{\mathrm{f}}\right)} \delta \mathbf{u} \cdot \boldsymbol{\xi} \mathrm{d} \mathbf{a} .
\end{aligned}
$$

The second term, $\mathrm{D}_{\mathbf{d}^{\mathrm{f}}} \mathcal{F}\left(\overline{\mathbf{u}}, \bar{p}, \overline{\mathbf{d}}^{\mathrm{f}} ; \overline{\mathbf{d}}^{\mathrm{s}}\right) \delta \mathbf{d}^{\mathrm{f}}$, requires the derivation with respect to $\mathbf{d}^{\mathrm{f}}$ of Eulerian integrals over $\Omega^{\mathrm{f}}\left(\mathbf{d}^{\mathrm{f}}\right)$ and its boundary. Actually, this kind of derivatives can be viewed as shape derivatives, see for instance [30]. Some elements on shape derivative calculus are given in appendix A and allow to establish the following identity,

$$
\begin{aligned}
& \left\langle\mathrm{D}_{\mathbf{d}^{\mathrm{f}}} \mathcal{F}\left(\overline{\mathbf{u}}, \bar{p}, \overline{\mathbf{d}^{\mathrm{f}}} ; \overline{\mathbf{d}^{\mathrm{s}}}\right) \delta \mathbf{d}^{\mathrm{f}},\left(\mathbf{v}^{\mathrm{f}}, q, \boldsymbol{\xi}, \boldsymbol{\lambda}\right)\right\rangle=\frac{\rho}{\Delta t} \int_{\Omega^{\mathrm{f}}\left(\overline{\mathbf{d}}^{\mathrm{f}}\right)}\left(\operatorname{div} \delta \mathbf{d}^{\mathrm{f}}\right) \overline{\mathbf{u}} \cdot \mathbf{v}^{\mathrm{f}} \mathrm{d} \mathbf{x} \\
& +\int_{\Omega^{\mathrm{f}}\left(\overline{\mathbf{d}}^{\mathrm{f}}\right)} \rho \operatorname{div}\left\{\overline{\mathbf{u}} \otimes\left(\mathbf{u}^{n}-\mathbf{w}_{\mathrm{g}}\left(\overline{\mathbf{d}^{\mathrm{f}}}\right)\right)\left[\operatorname{Idiv} \delta \mathbf{d}^{\mathrm{f}}-\left(\nabla \delta \mathbf{d}^{\mathrm{f}}\right)^{\mathrm{T}}\right]\right\} \cdot \mathbf{v}^{\mathrm{f}} \mathrm{d} \mathbf{x} \\
& -\frac{\rho}{\Delta t} \int_{\Omega^{\mathrm{f}}\left(\overline{\mathbf{d}}_{\mathrm{f}}^{\mathrm{f}}\right)} \operatorname{div}\left(\overline{\mathbf{u}} \otimes \delta \mathbf{d}^{\mathrm{f}}\right) \cdot \mathbf{v}^{\mathrm{f}} \mathrm{d} \mathbf{x}+\int_{\Omega^{\mathrm{f}}\left(\overline{\mathbf{d}}^{\mathrm{f}}\right)} \sigma(\overline{\mathbf{u}}, \bar{p})\left[\operatorname{I} \operatorname{div} \delta \mathbf{d}^{\mathrm{f}}-\left(\nabla \delta \mathbf{d}^{\mathrm{f}}\right)^{\mathrm{T}}\right]: \nabla \mathbf{v}^{\mathrm{f}} \mathrm{d} \mathbf{x} \\
& -\int_{\Omega^{\mathrm{f}}\left(\overline{\mathbf{d}}^{\mathrm{f}}\right)} \mu\left[\nabla \overline{\mathbf{u}} \nabla \delta \mathbf{d}^{\mathrm{f}}+\left(\nabla \delta \mathbf{d}^{\mathrm{f}}\right)^{\mathrm{T}}(\nabla \overline{\mathbf{u}})^{\mathrm{T}}\right]: \nabla \mathbf{v}^{\mathrm{f}} \mathrm{d} \mathbf{x}-\int_{\Gamma_{\text {in }-o u t}\left(\overline{\mathbf{d}}^{\mathrm{f}}\right)}\left(\boldsymbol{\eta}\left(\delta \mathbf{d}^{\mathrm{f}}\right) \cdot \mathbf{n}\right) \mathbf{g}\left(t_{n+1}\right) \cdot \mathbf{v}^{\mathrm{f}} \mathrm{d} \mathbf{a} \\
& -\int_{\Omega^{\mathrm{f}}\left(\overline{\mathbf{d}}^{\mathrm{f}}\right)} q \operatorname{div}\left\{\overline{\mathbf{u}}\left[\operatorname{Idiv} \delta \mathbf{d}^{\mathrm{f}}-\left(\nabla \delta \mathbf{d}^{\mathrm{f}}\right)^{\mathrm{T}}\right]\right\} \mathrm{d} \mathbf{x}+\int_{\Omega_{0}^{\mathrm{f}}} \delta \mathbf{d}^{\mathrm{f}} \cdot \boldsymbol{\lambda} \mathrm{d} \mathbf{x}_{0} \\
& +\int_{\Gamma^{\mathrm{w}}\left(\overline{\mathbf{d}}^{\mathrm{f}}\right)}\left[\left(\overline{\mathbf{u}}-\mathbf{w}_{\mathrm{g}}\left(\overline{\mathbf{d}^{\mathrm{f}}}\right)\right)\left(\boldsymbol{\eta}\left(\delta \mathbf{d}^{\mathrm{f}}\right) \cdot \mathbf{n}\right)-\frac{\left.\delta \mathbf{d}^{\mathrm{f}}\right] \cdot \boldsymbol{\xi} \mathrm{d} \mathbf{a} .}{\Delta t}\right]
\end{aligned}
$$

Here, we used the notation $\boldsymbol{\eta}\left(\delta \mathbf{d}^{\mathrm{f}}\right) \stackrel{\text { def }}{=}\left[\operatorname{Idiv} \delta \mathbf{d}^{\mathrm{f}}-\left(\nabla \delta \mathbf{d}^{\mathrm{f}}\right)^{\mathrm{T}}\right] \mathbf{n}$, which represents the first order variation of the surface vector $\mathbf{n}$ da (see, for instance, $[11,9]$ ). The derivative of $\mathcal{F}$ with respect to the solid variable is given by,

$$
\left\langle\mathrm{D}_{\mathbf{d}^{\mathrm{s}}} \mathcal{F}\left(\overline{\mathbf{u}}, \bar{p}, \overline{\mathbf{d}}^{\mathrm{f}} ; \overline{\mathbf{d}}^{\mathrm{s}}\right) \delta \mathbf{d}^{\mathrm{s}},\left(\mathbf{v}^{\mathrm{f}}, q, \boldsymbol{\xi}, \boldsymbol{\lambda}\right)\right\rangle=-\int_{\Omega_{0}^{\mathrm{f}}} \operatorname{Ext}^{\prime}\left(\overline{\mathbf{d}}^{\mathrm{s}}\right) \delta \mathbf{d}^{\mathrm{s}} \cdot \boldsymbol{\lambda} \mathrm{d} \mathbf{x}_{0}
$$

with the notation $\operatorname{Ext}^{\prime}\left(\overline{\mathbf{d}^{\mathrm{s}}}\right) \delta \mathbf{d}^{\mathrm{s}} \stackrel{\text { def }}{=} \mathrm{D}_{\mathbf{d}^{\mathrm{s}}} \operatorname{Ext}\left(\overline{\mathbf{d}^{\mathrm{s}}}\right) \delta \mathbf{d}^{\mathrm{s}}$. 


\subsection{Solid operator derivatives}

We proceed in the same fashion for the solid operator differentiation. We first get the classical Fréchet derivatives,

$$
\begin{aligned}
& \left\langle\mathrm{D}_{\mathbf{d}^{\mathrm{s}}} \mathcal{S}\left(\overline{\mathbf{u}}, \bar{p}, \overline{\mathbf{d}^{\mathrm{f}}} ; \mathbf{d}^{\mathrm{s}}\right) \delta \mathbf{d}^{\mathrm{s}}, \mathbf{v}^{\mathrm{s}}\right\rangle=\frac{2}{(\Delta t)^{2}} \int_{\Omega_{0}^{\mathrm{s}}} \rho_{0} \delta \mathbf{d}^{\mathrm{s}} \cdot \mathbf{v}^{\mathrm{s}} \mathrm{d} \mathbf{x}_{0} \\
& +\frac{1}{2} \int_{\Omega_{0}^{\mathrm{s}}}\left(F\left(\overline{\mathbf{d}^{\mathrm{s}}}\right) \delta S+\nabla \delta \mathbf{d}^{\mathrm{s}} S\left(\overline{\mathbf{d}^{\mathrm{s}}}\right)\right): \nabla \mathbf{v}^{\mathrm{s}} \mathrm{d} \mathbf{x}_{0}
\end{aligned}
$$

where $\delta S \stackrel{\text { def }}{=} \mathrm{D}_{\mathbf{d}^{\mathrm{s}}} S\left(\overline{\mathbf{d}^{\mathrm{s}}}\right) \delta \mathbf{d}^{\mathrm{s}}$.

Again we can split the derivative with respect to the fluid state in two terms:

$$
\begin{aligned}
\mathrm{D}_{\left(\mathbf{u}, p, \mathbf{d}^{\mathrm{f}}\right)} \mathcal{S}\left(\overline{\mathbf{u}}, \bar{p}, \overline{\mathbf{d}^{\mathrm{f}}} ; \overline{\mathbf{d}}^{\mathrm{s}}\right)\left(\delta \mathbf{u}, \delta p, \delta \mathbf{d}^{\mathrm{f}}\right)= & \mathrm{D}_{(\mathbf{u}, p)} \mathcal{S}\left(\overline{\mathbf{u}}, \bar{p}, \overline{\mathbf{d}}^{\mathrm{f}} ; \overline{\mathbf{d}}^{\mathrm{s}}\right)(\delta \mathbf{u}, \delta p) \\
& +\mathrm{D}_{\mathbf{d}^{\mathrm{f}}} \mathcal{S}\left(\overline{\mathbf{u}}, \bar{p}, \overline{\mathbf{d}^{\mathrm{f}}} ; \overline{\mathbf{d}}^{\mathrm{s}}\right) \delta \mathbf{d}^{\mathrm{f}} .
\end{aligned}
$$

The first term $\mathrm{D}_{(\mathbf{u}, p)} \mathcal{S}\left(\overline{\mathbf{u}}, \bar{p}, \overline{\mathbf{d}^{\mathrm{f}}} ; \overline{\mathbf{d}^{\mathbf{s}}}\right)(\delta \mathbf{u}, \delta p)$ is a classical Fréchet derivative. Thus, we have

$$
\begin{aligned}
& \left\langle\mathrm{D}_{(\mathbf{u}, p)} \mathcal{S}\left(\overline{\mathbf{u}}, \bar{p}, \overline{\mathbf{d}^{\mathrm{f}}} ; \overline{\mathbf{d}^{\mathrm{s}}}\right)(\delta \mathbf{u}, \delta p), \mathbf{v}^{\mathrm{s}}\right\rangle=\frac{\rho}{\Delta t} \int_{\Omega^{\mathrm{f}}\left(\overline{\mathbf{d}}^{\mathrm{f}}\right)} \delta \mathbf{u} \cdot \mathscr{R}\left(\mathbf{v}_{\mid \Gamma_{0}^{\mathrm{w}}}^{\mathrm{s}}\right) \mathrm{d} \mathbf{x} \\
& +\rho \int_{\Omega^{\mathrm{f}}\left(\overline{\mathbf{d}}^{\mathrm{f}}\right)} \operatorname{div}\left[\delta \mathbf{u} \otimes\left(\mathbf{u}^{n}-\mathbf{w}_{\mathrm{g}}\left(\overline{\mathbf{d}^{\mathrm{f}}}\right)\right] \cdot \mathscr{R}\left(\mathbf{v}_{\mid \Gamma_{0}^{\mathrm{w}}}^{\mathrm{s}}\right)+\int_{\Omega^{\mathrm{f}}\left(\overline{\mathbf{d}}^{\mathrm{f}}\right)} \sigma(\delta \mathbf{u}, \delta p): \nabla \mathscr{R}\left(\mathbf{v}_{\mid \Gamma_{0}^{\mathrm{w}}}^{\mathrm{s}}\right) \mathrm{d} \mathbf{x} .\right.
\end{aligned}
$$

Using the definition (9), the derivative of the solid operator with respect to $\mathbf{d}^{\mathrm{f}}$ corresponds to the derivative of the fluid loads operator with respect to $\mathbf{d}^{\mathrm{f}}$, i.e.

$$
\begin{aligned}
\left\langle\mathrm{D}_{\mathbf{d}^{\mathrm{f}}} \mathcal{S}\left(\overline{\mathbf{u}}, \bar{p}, \overline{\mathbf{d}^{\mathrm{f}}} ; \overline{\mathbf{d}^{\mathrm{s}}}\right) \delta \mathbf{d}^{\mathrm{f}}, \mathbf{v}^{\mathrm{s}}\right\rangle & =\left\langle\mathrm{D}_{\mathbf{d}^{\mathrm{f}}} \mathcal{L}\left(\overline{\mathbf{u}}, \bar{p}, \overline{\mathbf{d}^{\mathrm{f}}}\right) \delta \mathbf{d}^{\mathrm{f}}, \mathbf{v}^{\mathrm{s}}\right\rangle \\
& =\left\langle\mathrm{D}_{\mathbf{d}^{\mathrm{f}}} \mathcal{F}\left(\overline{\mathbf{u}}, \bar{p}, \overline{\mathbf{d}^{\mathrm{f}}} ; \overline{\mathbf{d}^{\mathrm{s}}}\right) \delta \mathbf{d}^{\mathrm{f}},\left(\mathscr{R}\left(\mathbf{v}_{\mid \Gamma_{0}^{\mathrm{w}}}^{\mathrm{s}}\right), 0, \mathbf{0}, \mathbf{0}\right)\right\rangle .
\end{aligned}
$$

Then using (18), we get

$$
\begin{aligned}
& \left\langle\mathrm{D}_{\mathbf{d}^{\mathrm{f}}} \mathcal{S}\left(\overline{\mathbf{u}}, \bar{p}, \overline{\mathbf{d}}^{\mathrm{f}} ; \overline{\mathbf{d}}^{\mathrm{s}}\right) \delta \mathbf{d}^{\mathrm{f}}, \mathbf{v}^{\mathrm{s}}\right\rangle=\frac{\rho}{\Delta t} \int_{\Omega^{\mathrm{f}}\left(\overline{\mathbf{d}}^{\mathrm{f}}\right)}\left(\operatorname{div} \delta \mathbf{d}^{\mathrm{f}}\right) \overline{\mathbf{u}} \cdot \mathscr{R}\left(\mathbf{v}_{\mid \Gamma_{0}^{\mathrm{w}}}^{\mathrm{s}}\right) \mathrm{d} \mathbf{x} \\
& +\int_{\Omega^{\mathrm{f}}\left(\overline{\mathbf{d}}^{\mathrm{f}}\right)} \rho \operatorname{div}\left\{\overline{\mathbf{u}} \otimes\left(\mathbf{u}^{n}-\mathbf{w}_{\mathrm{g}}\left(\overline{\mathbf{d}^{\mathrm{f}}}\right)\right)\left[\operatorname{Idiv} \delta \mathbf{d}^{\mathrm{f}}-\left(\nabla \delta \mathbf{d}^{\mathrm{f}}\right)^{\mathrm{T}}\right]\right\} \cdot \mathscr{R}\left(\mathbf{v}_{\mid \Gamma_{0}^{\mathrm{w}}}^{\mathrm{s}}\right) \mathrm{d} \mathbf{x} \\
& -\frac{\rho}{\Delta t} \int_{\Omega^{\mathrm{f}}\left(\overline{\mathbf{d}}^{\mathrm{f}}\right)} \operatorname{div}\left(\overline{\mathbf{u}} \otimes \delta \mathbf{d}^{\mathrm{f}}\right) \cdot \mathscr{R}\left(\mathbf{v}_{\mid \Gamma_{0}^{\mathrm{s}}}^{\mathrm{w}}\right) \mathrm{d} \mathbf{x} \\
& +\int_{\Omega^{\mathrm{f}}\left(\overline{\mathbf{d}}^{\mathrm{f}}\right)} \sigma(\overline{\mathbf{u}}, \bar{p})\left[\operatorname{I} \operatorname{div} \delta \mathbf{d}^{\mathrm{f}}-\left(\nabla \delta \mathbf{d}^{\mathrm{f}}\right)^{\mathrm{T}}\right]: \nabla \mathscr{R}\left(\mathbf{v}_{\mid \Gamma_{0}^{\mathrm{w}}}^{\mathrm{s}}\right) \mathrm{d} \mathbf{x} \\
& -\int_{\Omega^{\mathrm{f}}\left(\overline{\mathbf{d}}^{\mathrm{f}}\right)} \mu\left[\nabla \overline{\mathbf{u}} \nabla \delta \mathbf{d}^{\mathrm{f}}+\left(\nabla \delta \mathbf{d}^{\mathrm{f}}\right)^{\mathrm{T}}(\nabla \overline{\mathbf{u}})^{\mathrm{T}}\right]: \nabla \mathscr{R}\left(\mathbf{v}_{\mid \Gamma_{0}^{\mathrm{w}}}^{\mathrm{w}}\right) \mathrm{d} \mathbf{x},
\end{aligned}
$$

INRIA 


\section{Detailed Newton's algorithm sub-steps}

Let us recall the following notations:

$$
\begin{array}{rlr}
z \stackrel{\text { def }}{=} \mathbf{z}, & \bar{u}^{\mathrm{f}} \stackrel{\text { def }}{=}\left(\overline{\mathbf{u}}, \bar{p}, \overline{\mathbf{d}^{\mathrm{f}}}\right), & \bar{u}^{\mathrm{s}} \stackrel{\text { def }}{=} \overline{\mathbf{d}}^{\mathrm{s}}, \\
\hat{u}^{\mathrm{s}} \stackrel{\text { def }}{=} \hat{\mathbf{d}^{\mathrm{s}}}, & \delta u^{\mathrm{f}} \stackrel{\text { def }}{=}\left(\delta \mathbf{u}, \delta p, \delta \mathbf{d}^{\mathrm{f}}\right), & \delta z \stackrel{\text { def }}{=} \delta \mathbf{z} .
\end{array}
$$

Thus, from (16), it follows that step 2 (d) (i) consists in solving for $\left(\delta \mathbf{u}, \delta p, \delta \mathbf{d}^{\mathrm{f}}\right)$ the following linear variational problem

$$
\mathrm{D}_{(\mathbf{u}, p)} \mathcal{F}\left(\overline{\mathbf{u}}, \bar{p}, \overline{\mathbf{d}^{\mathrm{f}}} ; \overline{\mathbf{d}}^{\mathrm{s}}\right)(\delta \mathbf{u}, \delta p)+\mathrm{D}_{\mathbf{d}^{\mathrm{f}}} \mathcal{F}\left(\overline{\mathbf{u}}, \bar{p}, \overline{\mathbf{d}^{\mathrm{f}}} ; \overline{\mathbf{d}^{\mathrm{s}}}\right) \delta \mathbf{d}^{\mathrm{f}}=-\mathrm{D}_{\mathbf{d}^{\mathrm{f}}} \mathcal{F}\left(\overline{\mathbf{u}}, \bar{p}, \overline{\mathbf{d}^{\mathrm{f}}} ; \overline{\mathbf{d}^{\mathrm{s}}}\right)
$$

Using the expressions of the fluid operator derivatives provided in (17)-(19) and by taking $(\mathbf{0}, 0, \mathbf{0}, \boldsymbol{\lambda})$ as test function in $(24)$ we obtain that

$$
\delta \mathbf{d}^{\mathrm{f}}=\operatorname{Ext}^{\prime}\left(\overline{\mathbf{d}^{\mathrm{s}}}\right) \mathbf{z}, \quad \text { in } \quad \Omega_{0}^{\mathrm{f}} .
$$

On the other hand, taking the quadruplet $(\mathbf{0}, 0, \boldsymbol{\xi}, \mathbf{0})$ as test functions in $(24)$, we obtain the following boundary condition,

$$
\delta \mathbf{u}=\frac{\delta \mathbf{d}^{\mathrm{f}}}{\Delta t}-\left(\overline{\mathbf{u}}-\mathbf{w}_{\mathrm{g}}\left(\overline{\mathbf{d}^{\mathrm{f}}}\right)\right)\left(\boldsymbol{\eta}\left(\delta \mathbf{d}^{\mathrm{f}}\right) \cdot \mathbf{n}\right), \quad \text { on } \quad \Gamma^{\mathrm{w}}\left(\overline{\mathbf{d}^{\mathrm{f}}}\right) .
$$

Moreover, since by definition $\bar{u}^{\mathrm{f}}=\mathscr{F}\left(\bar{u}^{\mathrm{s}}\right)$, it follows that $\overline{\mathbf{u}}-\mathbf{w}_{\mathrm{g}}\left(\overline{\mathbf{d}^{\mathrm{f}}}\right)=0$ on $\Gamma^{\mathrm{w}}\left(\overline{\mathbf{d}^{\mathrm{f}}}\right)$ so that (25) reduces to

$$
\delta \mathbf{u}=\frac{\delta \mathbf{d}^{\mathrm{f}}}{\Delta t}, \quad \text { on } \quad \Gamma^{\mathrm{w}}\left(\overline{\mathbf{d}^{\mathrm{f}}}\right) .
$$

Finally, using (17)-(19) and choosing the quadruplet $(\mathbf{0}, 0, \boldsymbol{\xi}, \mathbf{0})$ as test functions in (24), we obtain the following variational expression satisfied by $(\delta \mathbf{u}, \delta p)$,

$$
\left\langle\mathrm{D}_{(\mathbf{u}, p)} \mathcal{F}\left(\overline{\mathbf{u}}, \bar{p}, \overline{\mathbf{d}}^{\mathrm{f}} ; \overline{\mathbf{d}}^{\mathrm{s}}\right)(\delta \mathbf{u}, \delta p),\left(\mathbf{v}^{\mathrm{f}}, q, \mathbf{0}, \mathbf{0}\right)\right\rangle=-\left\langle\mathrm{D}_{\mathbf{d}^{\mathrm{f}}} \mathcal{F}\left(\overline{\mathbf{u}}, \bar{p}, \overline{\mathbf{d}}^{\mathrm{f}} ; \overline{\mathbf{d}}^{\mathrm{s}}\right) \delta \mathbf{d}^{\mathrm{f}},\left(\mathbf{v}^{\mathrm{f}}, q, \mathbf{0}, \mathbf{0}\right)\right\rangle,
$$

Actually, the last expression corresponds to the weak formulation associated to the following PDE involving the linearized ALE Navier-Stokes equations:

$$
\left\{\begin{array}{l}
\frac{\rho}{\Delta t} \delta \mathbf{u}+\rho \operatorname{div}\left[\delta \mathbf{u} \otimes\left(\mathbf{u}^{n}-\mathbf{w}_{\mathrm{g}}\left(\overline{\mathbf{d}}^{\mathrm{f}}\right)\right)-\sigma(\delta \mathbf{u}, \delta p)\right]=-\frac{\rho}{\Delta t}\left(\operatorname{div} \delta \mathbf{d}^{\mathrm{f}}\right) \overline{\mathbf{u}} \\
\quad-\operatorname{div}\left\{\left[\rho \overline{\mathbf{u}} \otimes\left(\mathbf{u}^{n}-\mathbf{w}_{\mathrm{g}}\left(\overline{\mathbf{d}}^{\mathrm{f}}\right)\right)-\sigma(\overline{\mathbf{u}}, \bar{p})\right]\left[\operatorname{Idiv} \delta \mathbf{d}^{\mathrm{f}}-\left(\nabla \delta \mathbf{d}^{\mathrm{f}}\right)^{\mathrm{T}}\right]\right\} \\
\quad+\frac{\rho}{\Delta t} \operatorname{div}\left(\overline{\mathbf{u}} \otimes \delta \mathbf{d}^{\mathrm{f}}\right)-\operatorname{div}\left\{\mu\left[\nabla \overline{\mathbf{u}} \nabla \delta \mathbf{d}^{\mathrm{f}}+\left(\nabla \delta \mathbf{d}^{\mathrm{f}}\right)^{\mathrm{T}}(\nabla \overline{\mathbf{u}})^{\mathrm{T}}\right]\right\}, \quad \text { in } \quad \Omega^{\mathrm{f}}(\overline{\mathbf{d}}), \\
\operatorname{div} \delta \mathbf{u}=-\operatorname{div}\left\{\overline{\mathbf{u}}\left[\operatorname{I} \operatorname{div} \delta \mathbf{d}^{\mathrm{f}}-\left(\nabla \delta \mathbf{d}^{\mathrm{f}}\right)^{\mathrm{T}}\right]\right\}, \quad \text { in } \quad \Omega^{\mathrm{f}}\left(\overline{\mathbf{d}}^{\mathrm{f}}\right),
\end{array}\right.
$$

$\mathrm{RR} \mathrm{n}^{\circ} 5087$ 
endowed with the following inflow-outflow condition on $\Gamma_{\text {in-out }}\left(\overline{\mathbf{d}}^{\mathrm{f}}\right)$ :

$$
\sigma(\delta \mathbf{u}, \delta p) \mathbf{n}=\mathbf{g}\left(t_{n+1}\right)\left(\boldsymbol{\eta}\left(\delta \mathbf{d}^{\mathrm{f}}\right) \cdot \mathbf{n}\right)-\sigma(\overline{\mathbf{u}}, \bar{p}) \boldsymbol{\eta}\left(\delta \mathbf{d}^{\mathrm{f}}\right)+\mu\left[\nabla \overline{\mathbf{u}} \nabla \delta \mathbf{d}^{\mathrm{f}}+\left(\nabla \delta \mathbf{d}^{\mathrm{f}}\right)^{\mathrm{T}}(\nabla \overline{\mathbf{u}})^{\mathrm{T}}\right] \mathbf{n} .
$$

In short, taking into account the above considerations, step 2(d) (i) can be carried out by computing $\delta \mathbf{d}^{\mathrm{f}}=\operatorname{Ext}^{\prime}\left(\overline{\mathbf{d}}^{\mathrm{s}}\right) \mathbf{z}$ and by solving the following linearized Navier-Stokes problem for $(\delta \mathbf{u}, \delta p)$ :

$$
\left\{\begin{array}{l}
\frac{\rho}{\Delta t} \delta \mathbf{u}+\rho \operatorname{div}\left[\delta \mathbf{u} \otimes\left(\mathbf{u}^{n}-\mathbf{w}_{\mathrm{g}}\left(\overline{\mathbf{d}^{\mathrm{f}}}\right)\right)-\sigma(\delta \mathbf{u}, \delta p)\right]=-\frac{\rho}{\Delta t}\left(\operatorname{div} \delta \mathbf{d}^{\mathrm{f}}\right) \overline{\mathbf{u}} \\
\quad-\operatorname{div}\left\{\left[\rho \overline{\mathbf{u}} \otimes\left(\mathbf{u}^{n}-\mathbf{w}_{\mathrm{g}}\left(\overline{\mathbf{d}^{\mathrm{f}}}\right)\right)-\sigma(\overline{\mathbf{u}}, \bar{p})\right]\left[\operatorname{Idiv} \delta \mathbf{d}^{\mathrm{f}}-\left(\nabla \delta \mathbf{d}^{\mathrm{f}}\right)^{\mathrm{T}}\right]\right\} \\
\quad+\frac{\rho}{\Delta t} \operatorname{div}\left(\overline{\mathbf{u}} \otimes \delta \mathbf{d}^{\mathrm{f}}\right)-\operatorname{div}\left\{\mu\left[\nabla \overline{\mathbf{u}} \nabla \delta \mathbf{d}^{\mathrm{f}}+\left(\nabla \delta \mathbf{d}^{\mathrm{f}}\right)^{\mathrm{T}}(\nabla \overline{\mathbf{u}})^{\mathrm{T}}\right]\right\}, \quad \text { in } \quad \Omega^{\mathrm{f}}(\overline{\mathbf{d}}), \\
\operatorname{div} \delta \mathbf{u}=-\operatorname{div}\left\{\overline{\mathbf{u}}\left[\operatorname{I} \operatorname{div} \delta \mathbf{d}^{\mathrm{f}}-\left(\nabla \delta \mathbf{d}^{\mathrm{f}}\right)^{\mathrm{T}}\right]\right\}, \quad \text { in } \quad \Omega^{\mathrm{f}}\left(\overline{\mathbf{d}^{\mathrm{f}}}\right), \\
\delta \mathbf{u}=\frac{\delta \mathbf{d}^{\mathrm{f}}}{\Delta t}, \quad \text { on } \quad \Gamma^{\mathrm{w}}\left(\overline{\mathbf{d}^{\mathrm{f}}}\right), \\
\sigma(\delta \mathbf{u}, \delta p) \mathbf{n}=\mathbf{g}\left(t_{n+1}\right)\left(\boldsymbol{\eta}\left(\delta \mathbf{d}^{\mathrm{f}}\right) \cdot \mathbf{n}\right)-\sigma(\overline{\mathbf{u}}, \bar{p}) \boldsymbol{\eta}\left(\delta \mathbf{d}^{\mathrm{f}}\right) \\
\quad+\mu\left[\nabla \overline{\mathbf{u}} \nabla \delta \mathbf{d}^{\mathrm{f}}+\left(\nabla \delta \mathbf{d}^{\mathrm{f}}\right)^{\mathrm{T}}(\nabla \overline{\mathbf{u}})^{\mathrm{T}}\right] \mathbf{n}, \quad \text { on } \quad \Gamma_{\text {in }- \text { out }}\left(\overline{\mathbf{d}^{\mathrm{f}}}\right)
\end{array}\right.
$$

Finally, using the expressions of the solid operator derivatives provided in (20)-(23), we are able to identify the structure of step 2 (d) (ii). It consists in solving for the perturbed solid state $\delta \mathbf{z}$ the following problem:

$$
\begin{aligned}
& \frac{2}{(\Delta t)^{2}} \int_{\Omega_{0}^{\mathrm{s}}} \rho_{0} \delta \mathbf{z} \cdot \mathbf{v}^{\mathrm{s}} \mathrm{d} \mathbf{x}_{0}+\frac{1}{2} \int_{\Omega_{0}^{\mathrm{s}}}\left(F\left(\hat{\mathbf{d}}^{\mathrm{s}}\right) \delta S(\mathbf{z})+\nabla \delta \mathbf{z} S\left(\hat{\mathbf{d}}^{\mathrm{s}}\right)\right): \nabla \mathbf{v}^{\mathrm{s}} \mathrm{d} \mathbf{x}_{0} \\
& =\frac{\rho}{\Delta t} \int_{\Omega^{\mathrm{f}}\left(\overline{\mathbf{d}}^{\mathrm{f}}\right)} \delta \mathbf{u} \cdot \mathscr{R}\left(\mathbf{v}_{\mid \Gamma_{0}^{\mathrm{w}}}^{\mathrm{s}}\right) \mathrm{d} \mathbf{x}+\rho \int_{\Omega^{\mathrm{f}}\left(\overline{\mathbf{d}}^{\mathrm{f}}\right)} \operatorname{div}\left[\delta \mathbf{u} \otimes\left(\mathbf{u}^{n}-\mathbf{w}_{\mathrm{g}}\left(\overline{\mathbf{d}}^{\mathrm{f}}\right)\right] \cdot \mathscr{R}\left(\mathbf{v}_{\mid \Gamma_{0}^{\mathrm{w}}}^{\mathrm{s}}\right)\right. \\
& +\int_{\Omega^{\mathrm{f}}\left(\overline{\mathbf{d}^{\mathrm{f}}}\right)} \sigma(\delta \mathbf{u}, \delta p): \nabla \mathscr{R}\left(\mathbf{v}_{\mid \Gamma_{0}^{\mathrm{w}}}^{\mathrm{s}}\right) \mathrm{d} \mathbf{x}+\frac{\rho}{\Delta t} \int_{\Omega^{\mathrm{f}}\left(\overline{\mathbf{d}^{\mathrm{f}}}\right)}\left(\operatorname{div} \delta \mathbf{d}^{\mathrm{f}}\right) \overline{\mathbf{u}} \cdot \mathscr{R}\left(\mathbf{v}_{\mid \Gamma_{0}^{\mathrm{w}}}^{\mathrm{s}}\right) \mathrm{d} \mathbf{x} \\
& +\int_{\Omega^{\mathrm{f}}\left(\overline{\mathbf{d}}^{\mathrm{f}}\right)} \rho \operatorname{div}\left\{\overline{\mathbf{u}} \otimes\left(\mathbf{u}^{n}-\mathbf{w}_{\mathrm{g}}\left(\overline{\mathbf{d}^{\mathrm{f}}}\right)\right)\left[\mathrm{I} \operatorname{div} \delta \mathbf{d}^{\mathrm{f}}-\left(\nabla \delta \mathbf{d}^{\mathrm{f}}\right)^{\mathrm{T}}\right]\right\} \cdot \mathscr{R}\left(\mathbf{v}_{\mid \Gamma_{0}^{\mathrm{w}}}^{\mathrm{s}}\right) \mathrm{d} \mathbf{x} \\
& -\frac{\rho}{\Delta t} \int_{\Omega^{\mathrm{f}}\left(\overline{\mathbf{d}^{\mathrm{f}}}\right)} \operatorname{div}\left(\overline{\mathbf{u}} \otimes \delta \mathbf{d}^{\mathrm{f}}\right) \cdot \mathscr{R}\left(\mathbf{v}_{\mid \Gamma_{0}^{\mathrm{w}}}^{\mathrm{s}}\right) \mathrm{d} \mathbf{x} \\
& +\int_{\Omega^{\mathrm{f}}\left(\overline{\mathbf{d}}^{\mathrm{f}}\right)} \sigma(\overline{\mathbf{u}}, \bar{p})\left[\mathrm{I} \operatorname{div} \delta \mathbf{d}^{\mathrm{f}}-\left(\nabla \delta \mathbf{d}^{\mathrm{f}}\right)^{\mathrm{T}}\right]: \nabla \mathscr{R}\left(\mathbf{v}_{\mid \Gamma_{0}^{\mathrm{w}}}^{\mathrm{w}}\right) \mathrm{d} \mathbf{x} \\
& -\int_{\Omega^{\mathrm{f}}\left(\overline{\mathbf{d}}^{\mathrm{f}}\right)} \mu\left[\nabla \overline{\mathbf{u}} \nabla \delta \mathbf{d}^{\mathrm{f}}+\left(\nabla \delta \mathbf{d}^{\mathrm{f}}\right)^{\mathrm{T}}(\nabla \overline{\mathbf{u}})^{\mathrm{T}}\right]: \nabla \mathscr{R}\left(\mathbf{v}_{\mid \Gamma_{0}^{\mathrm{w}}}^{\mathrm{s}}\right) \mathrm{d} \mathbf{x},
\end{aligned}
$$

INRIA 
Let notice, that the right hand side of this problem is nothing but the residual of the linearized fluid momentum equation $(28)_{1}$ involved in step 2 (d) (i).

An approximate fluid tangent problem can be simply derived from exact expression (28) by neglecting the shape derivative terms (see $[31,5,7]$ ), yielding

$$
\left\{\begin{array}{l}
\frac{\rho}{\Delta t} \delta \mathbf{u}+\rho \operatorname{div}\left[\delta \mathbf{u} \otimes\left(\mathbf{u}^{n}-\mathbf{w}_{\mathrm{g}}\left(\overline{\mathbf{d}}^{\mathrm{f}}\right)\right)-\sigma(\delta \mathbf{u}, \delta p)\right]=\mathbf{0}, \quad \text { in } \Omega^{\mathrm{f}}\left(\overline{\mathbf{d}}^{\mathrm{f}}\right), \\
\operatorname{div} \delta \mathbf{u}=0, \quad \text { in } \quad \Omega^{\mathrm{f}}\left(\overline{\mathbf{d}}^{\mathrm{f}}\right), \\
\delta \mathbf{u}=\frac{\delta \mathbf{d}^{\mathrm{f}}}{\Delta t}, \quad \text { on } \quad \Gamma^{\mathrm{w}}\left(\overline{\mathbf{d}^{\mathrm{f}}}\right), \\
\sigma(\delta \mathbf{u}, \delta p) \mathbf{n}=0, \quad \text { on } \quad \Gamma_{\text {in-out }}\left(\overline{\mathbf{d}^{\mathrm{f}}}\right),
\end{array}\right.
$$

Still, by neglecting here the convective and diffusive terms we get the following approximate fluid tangent problem:

$$
\left\{\begin{array}{l}
\frac{\rho}{\Delta t} \delta \mathbf{u}+\nabla \delta p=\mathbf{0}, \quad \text { in } \quad \Omega^{\mathrm{f}}\left(\overline{\mathbf{d}}^{\mathrm{f}}\right) \\
\operatorname{div} \delta \mathbf{u}=0, \quad \text { in } \quad \Omega^{\mathrm{f}}\left(\overline{\mathbf{d}^{\mathrm{f}}}\right) \\
\delta \mathbf{u}=\frac{\delta \mathbf{d}^{\mathrm{f}}}{\Delta t}, \quad \text { on } \quad \Gamma^{\mathrm{w}}\left(\overline{\mathbf{d}^{\mathrm{f}}}\right) \\
\delta p \mathbf{n}=\mathbf{0}, \quad \text { on } \quad \Gamma_{\text {in }- \text { out }}\left(\overline{\mathbf{d}^{\mathrm{f}}}\right)
\end{array}\right.
$$

that was introduced in $[16]$ (see also $[7,17]$ ).

\section{Numerical results}

In this section we compare the exact Newton's algorithm, whose jacobian evaluations have been described in Section 7, with two former approaches:

- fixed-point iterations combined with an Aitken's acceleration techniques (FP-Aitken) $[25,16]$,

- a quasi-Newton's algorithm, whose approximate jacobians evaluations are carried out using the simplified fluid tangent problem (29), i.e., without taking into account the shape derivatives of the fluid operator (see $[31,5,7]$ ).

As we shall see, in the sequel, the expected superiority of the exact jacobian approach is validated by the numerical experiments, mainly when using moderate time steps.

We consider a fluid-structure problem arising in the modelling of the blood flow in large arteries: it consists of a thin elastic vessel conveying an incompressible viscous fluid (see $[6,5,28,14,16])$. The solid is described by the linear elasticity equations (Saint Venant-Kirchhof material), while the fluid is described by the incompressible Navier-Stokes equations. We consider two different geometries:

1. a straight cylinder of radius $R_{0}=0.5 \mathrm{~cm}$ and length $L=5 \mathrm{~cm}$, 


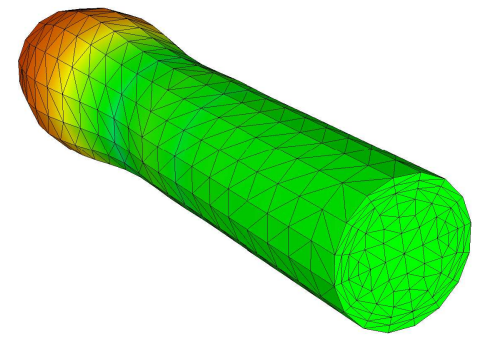

(a) $t=0.0025 \mathrm{~s}$



(c) $t=0.0075 \mathrm{~s}$

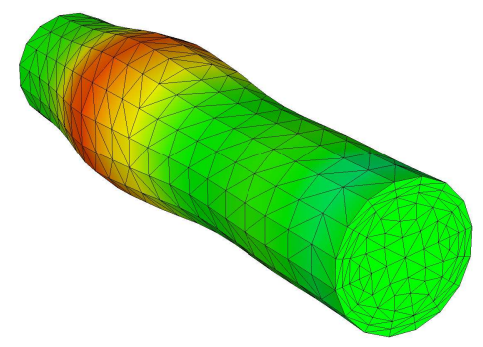

(b) $t=0.005 \mathrm{~s}$

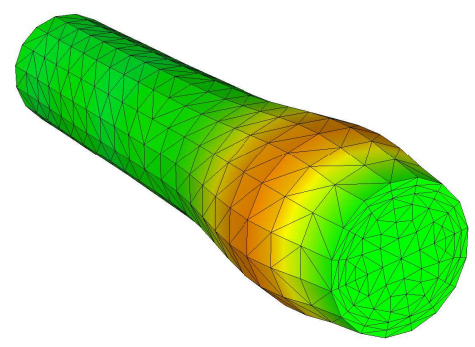

(d) $t=0.01 \mathrm{~s}$

Figure 3: Pressure wave propagation (straight cylinder)

2. a curved cylinder of radius $R_{0}=0.5 \mathrm{~cm}$ with curvature ratio $0.25 \mathrm{~cm}^{-1}$.

The surrounding structure has a thickness of $h=0.1 \mathrm{~cm}$. The physical parameters are the following (see $[14,16])$ :

- Fluid: viscosity $\mu=0.003$ poise, density $\rho=1 \mathrm{~g} / \mathrm{cm}^{3}$,

- Solid: density $\rho_{0}=1.2 \mathrm{~g} / \mathrm{cm}^{3}$, Young modulus $E=3 \times 10^{6}$ dynes $/ \mathrm{cm}^{2}$ and Poisson ratio $\nu=0.3$. 


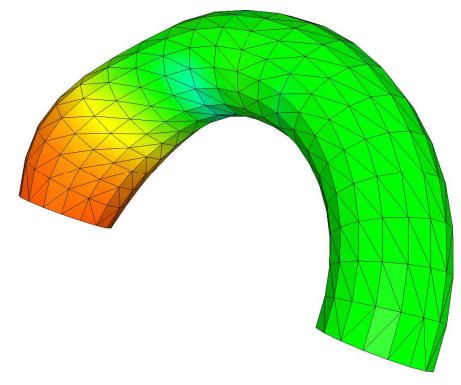

(a) $t=0.0025 \mathrm{~s}$

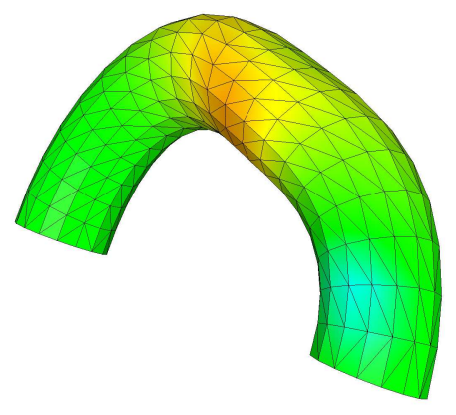

(c) $t=0.0075 \mathrm{~s}$

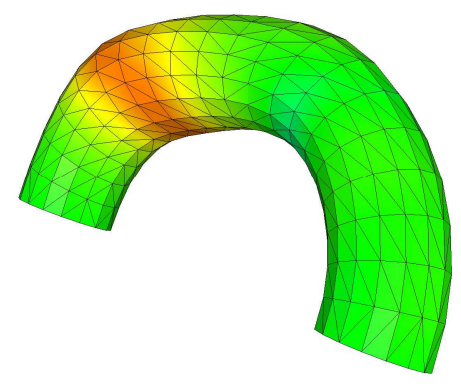

(b) $t=0.005 \mathrm{~s}$

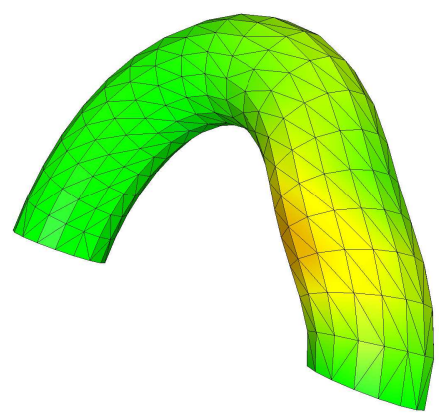

(d) $t=0.01 \mathrm{~s}$

Figure 4: Pressure wave propagation (curved cylinder)

Both systems, the fluid and the structure, are initially at rest. The structure is clamped at the inlet and the outlet. An over pressure of $1.3332 \times 10^{-4}$ dynes $/ \mathrm{cm}^{2}(10 \mathrm{mmHg})$ is imposed on the inlet boundary $\Gamma_{\text {in }}$ during $3 \times 10^{-3}$ seconds. The fluid equations are discretised using $\mathbb{P}_{1}$ bubble $/ \mathbb{P}_{1}$ finite elements, whereas for the solid equations we use $\mathbb{P}_{1}$ finite elements.

A pressure wave propagation is observed in both configurations. Figures 3 and 4 show the fluid pressure at time $t=0.0025,0.005,0.0075,0.01 \mathrm{~s}$ with time step $\Delta t=10^{-4} \mathrm{~s}$. These results are similar to those provided in $[16,14]$. In figures 5 and 6 the corresponding solid deformed configurations (half section) are displayed. 
Remark 4 The boundary data imposed on the inlet and outlet boundaries [14, 16, 17] do not have any physiological meaning. Let us notice that the typical period of a heart beat is about 1 second [27]. Providing realistic physiological simulations of the interaction between the blood and the arterial wall, lies outside the scope of this paper. This will be the purpose of a forthcoming work. In such cases, the use of moderate time steps will be crucial to perform over several cardiac beats.

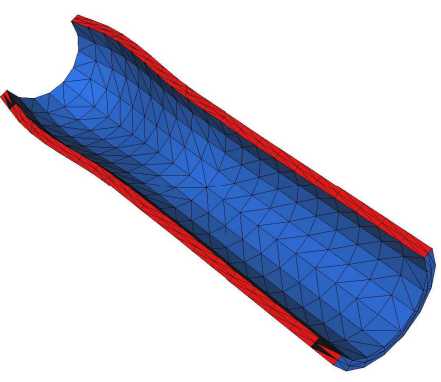

(a) $t=0.0025 \mathrm{~s}$

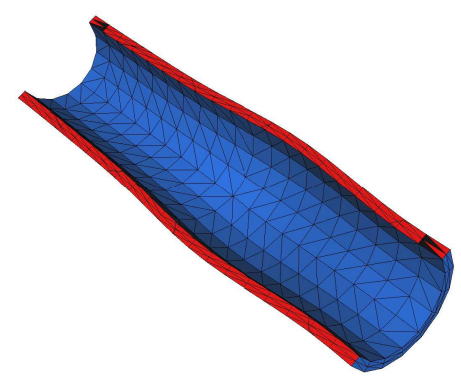

(c) $t=0.0075 \mathrm{~s}$

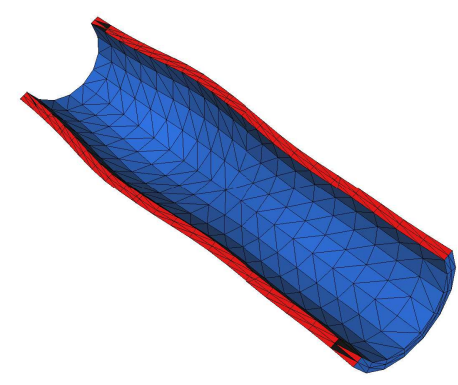

(b) $t=0.005 \mathrm{~s}$



(d) $t=0.01 \mathrm{~s}$

Figure 5: Solid domain deformed configuration (straight cylinder) 


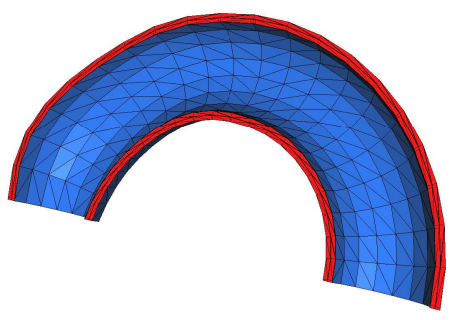

(a) $t=0.0025 \mathrm{~s}$



(c) $t=0.0075 \mathrm{~s}$

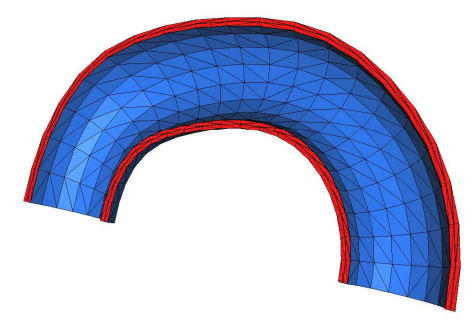

(b) $t=0.005 \mathrm{~s}$

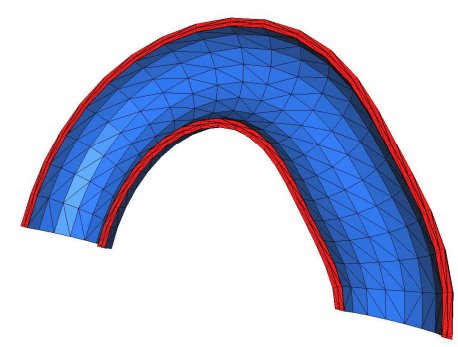

(d) $t=0.01 \mathrm{~s}$

Figure 6: Solid domain deformed configuration (curved cylinder)

For comparison purposes, we give in figures 7 and 8 the number of iterations of either method at each time level, for $\Delta t=10^{-4} \mathrm{~s}$. The superior convergence properties of both Newton's algorithms are evident. However, the cost of each Newton iteration is higher than the cost of a fixed-point iteration. Therefore, the previous promising convergence behavior does not necessarily imply an overall reduction of the computational cost. Hence, in order to compare the computational cost of these algorithms, we furnish in tables 1 and 2 the elapsed CPU time (dimensionless) for either algorithm after 300 time steps of length $\Delta t=10^{-4} \mathrm{~s}$. We can notice that both Newton's algorithms are (in our implementation) about 2 times 
faster than the fixed-point algorithm. This computational gain can be also recovered (from figures 7 and 8) by simply estimating the cost of each Newton iteration in terms of the number of fluid and solid solvers calls. Each Newton iteration requires:

- a fluid solver evaluation,

- a solid solver evaluation,

- about 9 inner GMRES iterations (with a tolerance of $10^{-4}$ ).

At this point, it is important to remark that each call of the fluid solver updates the fluid mesh and the corresponding FE fluid matrices, while the tangent fluid solver keeps the same FE matrix during the GMRES process. As a result, and since we are dealing with linear solvers, the cost of each Newton iteration is approximately 7 times the cost of a fluid solver evaluation, plus 10 times the cost of a solid solver evaluation. This explains the observed overall computational cost reduction. Obviously, this performance rises much more when the fluid and solid solvers are non-linear.

Now, let us address in details the comparison between the exact Newton and quasiNewton algorithms. Obviously, the GMRES iterations in the exact version are more expensive than in the quasi version. Indeed, the exact Newton's algorithm involves the computation of a shape derivative which is required by the fluid tangent problem (28). However, this is usually compensated by a reduced number of Newton iterations. For instance, Figures 7 and 8 and tables 1 and 2 show that, for $\Delta t=10^{-4}$ seconds, these algorithms exhibit a comparable behavior.

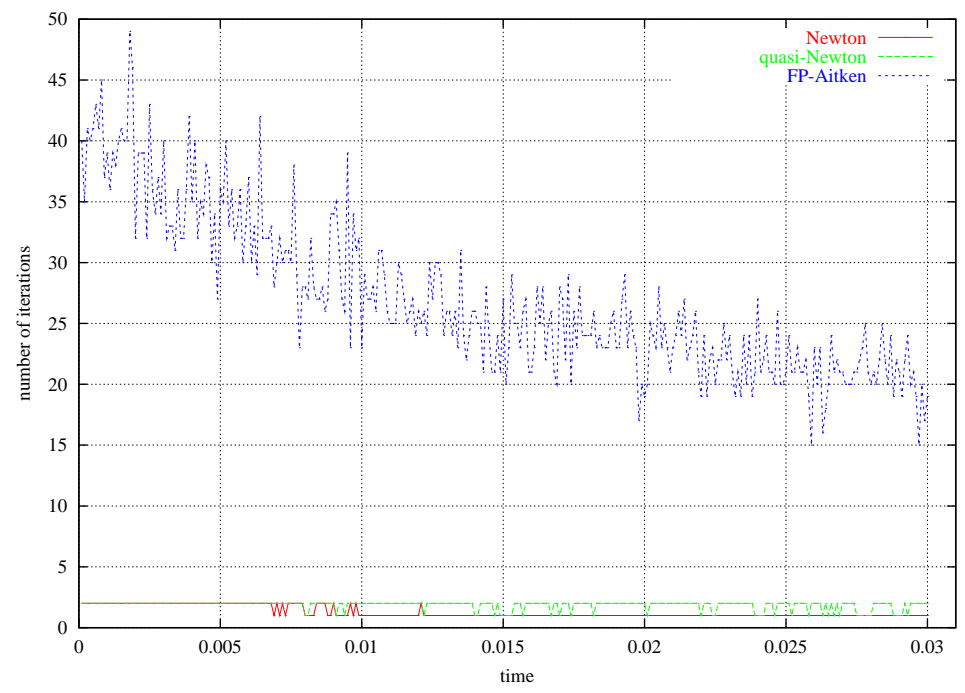

Figure 7: Straight cylinder $\Delta t=10^{-4} \mathrm{~s}$ 


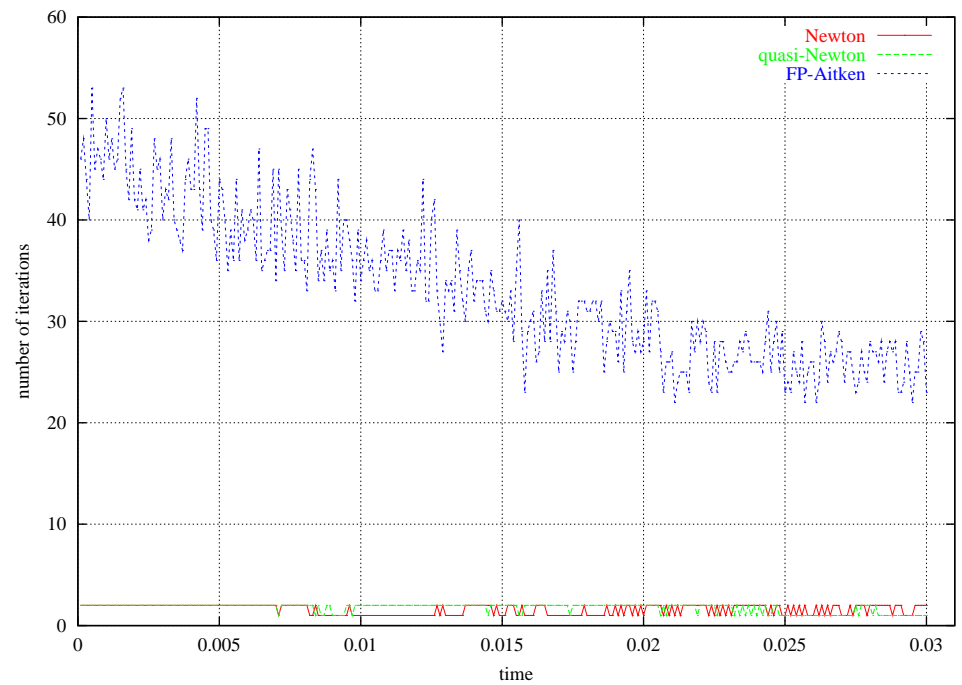

Figure 8: Curved cylinder $\Delta t=10^{-4} \mathrm{~s}$

\begin{tabular}{|c|c|}
\hline Algorithm & CPU time \\
\hline FP-Aitken & 1.00 \\
\hline Quasi-Newton & 0.56 \\
\hline Newton & 0.52 \\
\hline
\end{tabular}

Table 1: Dimensionless CPU time (straight cylinder)

\begin{tabular}{|c|c|}
\hline Algorithm & CPU time \\
\hline FP-Aitken & 1.00 \\
\hline Quasi-Newton & 0.55 \\
\hline Newton & 0.60 \\
\hline
\end{tabular}

Table 2: Dimensionless CPU time (curved cylinder)

The superiority of the exact Newton's algorithm can be simply illustrated by increasing the time step. Indeed, in figures 9 and 10 , we specify the number of iterations of either method at each time level, with time step $\Delta t=10^{-3} \mathrm{~s}$. The fixed-point and quasi-Newton algorithms fail to converge after two time steps: the allowed maximum number of iterations is in both cases reached, whereas the exact Newton's method converges and requires a low (almost constant) number of iterations. This illustrates the expected good convergence properties of the Newton's method using exact jacobians evaluations. Figures 11 and 12 show the evolution of the residual during the iteration process in both Newton's algorithms 


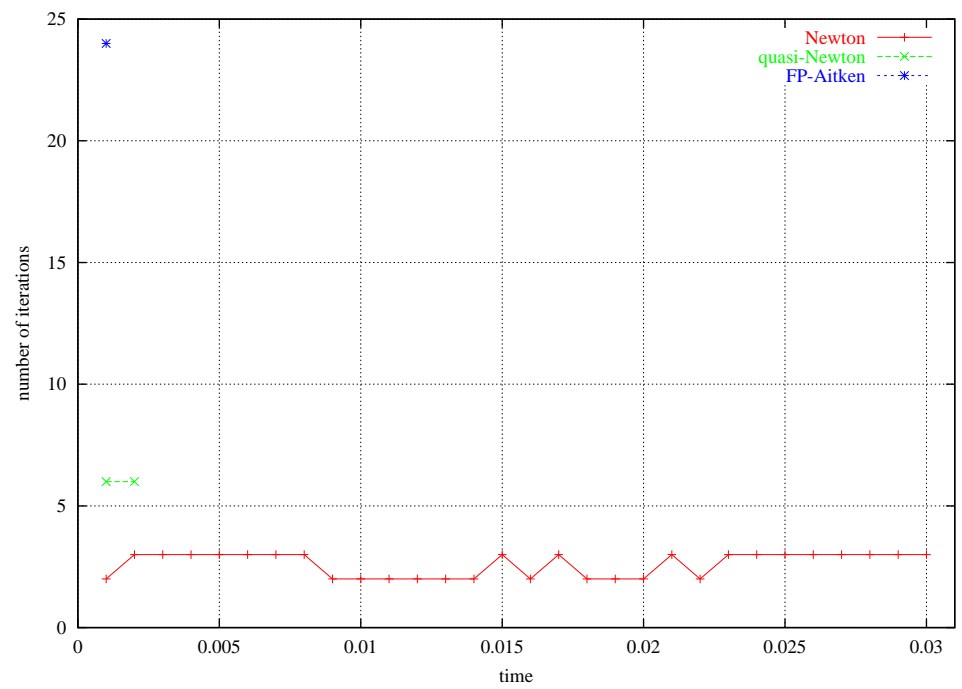

Figure 9: Straight cylinder $\Delta t=10^{-3} \mathrm{~s}$

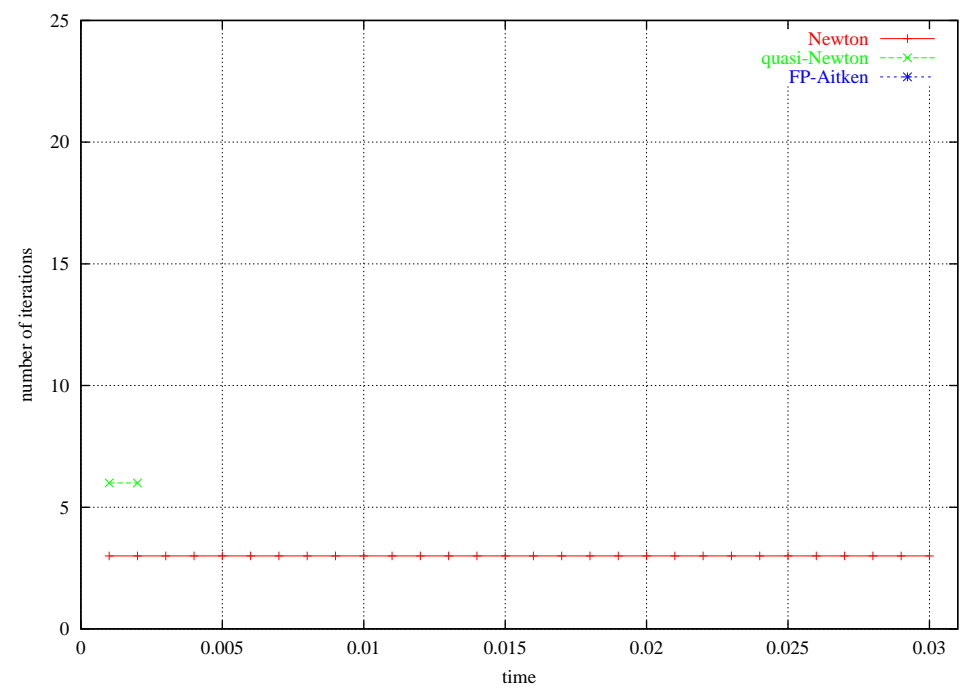

Figure 10: Curved cylinder $\Delta t=10^{-3} \mathrm{~s}$

at the third time step. We may observe that the quasi-Newton's algorithm is unable to 
reduce the residual after 100 iterations, while the exact Newton only requires 3 iterations to reach the convergence threshold.



Figure 11: Straight cylinder $\Delta t=10^{-3} \mathrm{~s}$



Figure 12: Curved cylinder $\Delta t=10^{-3} \mathrm{~s}$

$\mathrm{RR} \mathrm{n}^{\circ} 5087$ 
Let notice that the initial guess used in our computations, for starting each Newton's loop, is based on a extrapolation of the displacement of the previous time steps (see, for instance, $[22,28,16])$. Consequently, larger time steps lead to worse initial guess and then to a higher number of iterations. The variations of the fluid domain become thus more relevant and, therefore, the shape derivatives (18) can not be neglected in (28).

\section{Conclusion}

Using Newton based methods for solving the non-linear coupled systems arising in the numerical approximation of fluid-structure interaction problems, requires evaluations of the jacobian associated to coupled non-linear problem. Up to now, these evaluations were approximated either using finite differences [23, 24, 31, 19], or by introducing simplified expressions $[31,16,5,7,17]$. In any case, with a loss of some of the convergence features of the Newton's method. In this article, we have proposed a new strategy consisting in implementing linearized solvers in order to evaluate, in a consistent way, the different jacobians involved in the Newton's algorithm. The main contribution of this paper consists in establishing the expressions of these jacobians, in the case of a coupled system consisting of an incompressible Newtonian fluid interacting with a non-linear elastic solid under large displacements. On the numerical view point, the Newton's method has been implemented in a 3D research code in the case of a linear elastic solid. Performing several numerical experiments allowed us to show how the exactness level of the jacobian evaluations influences drastically the convergence properties of the Newton's loop.

There exist several ways of improving the results presented in this paper. First we shall move from a linear to a nonlinear elastic solid. Then we may address the technical point consisting in preconditioning the GMRES iterations during the Newton's loop [19, 7]. We shall also investigate the application of our approach in the case of disretisations involving space-time finite elements [31]. Our underlying motivation is, in any case, to be able to provide fast and reliable simulations of the mechanical interaction between the blood and the arterial wall $[14,28,6,5,16,17]$, over several cardiac beats.

\section{Acknowledgments}

First author was supported by the Swiss National Science Foundation (contract number 2065110.01). Second author was supported by the Semester Program "Mathematical Modelling of the Cardiovascular System" of the Bernoulli Center at the EPFL.

\section{A Shape derivative calculus}

We consider a smooth domain $\Omega_{0} \in \mathbb{R}^{3}$ and a one-to-one mapping $\mathbf{x} \in \mathcal{T}^{k, \infty}$ where

$$
\mathcal{T}^{k, \infty} \stackrel{\text { def }}{=}\left\{\mathbf{x} \quad \mid \quad \mathbf{x}-\mathrm{I} \in W^{k, \infty}\left(\mathbb{R}^{3}, \mathbb{R}^{3}\right), \quad \mathbf{x}^{-1}-\mathrm{I} \in W^{k, \infty}\left(\mathbb{R}^{3}, \mathbb{R}^{3}\right)\right\}
$$


with $k \geq 1$.

We introduce the transported domain $\Omega(\mathbf{x})=\mathbf{x}\left(\Omega_{0}\right)$. We introduce a scalar parameter $\tau \geq 0$ and a perturbation mapping $\delta \mathbf{x} \in \mathcal{T}^{k, \infty}$ and we set $F_{\tau}=\nabla(\mathbf{x}+\tau \delta \mathbf{x})$ and $J_{\tau}=\operatorname{det} F_{\tau}$. The following identities are easy to verify,

Lemma 1

$$
\begin{gathered}
\left.\mathrm{D}_{\mathbf{x}}[J] \delta \mathbf{x} \stackrel{\text { def }}{=} \frac{d}{d \tau} J_{\tau}\right|_{\tau=0}=J \operatorname{div} \delta \mathbf{x} \\
\mathrm{D}_{\mathbf{x}}\left[F^{-1}\right] \delta \mathbf{x}=-F^{-1} \nabla \delta \mathbf{x}
\end{gathered}
$$

We introduce the unit outward normal to $\Gamma(\mathbf{x}) \stackrel{\text { def }}{=} \partial \Omega(\mathbf{x})$ by

$$
\mathbf{n}(\mathbf{x})=\frac{F^{-T} \mathbf{n}_{0}}{\left\|F^{-T} \mathbf{n}_{0}\right\|}
$$

where $\mathbf{n}_{0}$ is unit outward normal to $\Gamma_{0} \stackrel{\text { def }}{=} \partial \Omega_{0}$. We have the following identities,

\section{Lemma 2}

$$
\begin{aligned}
& \mathrm{D}_{\mathbf{x}}\left[\int_{\Gamma(\mathbf{x})} \mathrm{d} \mathbf{a}\right] \delta \mathbf{x}=\int_{\Gamma(\mathbf{x})} \boldsymbol{\eta}(\delta \mathbf{x}) \cdot \mathbf{n} \mathrm{d} \mathbf{a} \\
& \mathrm{D}_{\mathbf{x}}\left[\int_{\Gamma(\mathbf{x})} \mathbf{n} \mathrm{d} \mathbf{a}\right] \delta \mathbf{x}=\int_{\Gamma(\mathbf{x})} \boldsymbol{\eta}(\delta \mathbf{x}) \mathrm{d} \mathbf{a}
\end{aligned}
$$

with $\boldsymbol{\eta}(\delta \mathbf{x})=\left[\mathrm{I} \operatorname{div} \delta \mathbf{x}-(\nabla \delta \mathbf{x})^{\mathrm{T}}\right] \mathbf{n}$

We also have the classical Piola identity,

\section{Lemma 3}

$$
\operatorname{div}\left[\operatorname{Idiv} \mathbf{x}-(\nabla \mathbf{x})^{\mathrm{T}}\right]=0
$$

Now we consider a function $\mathbf{u}(\mathbf{x}): \Omega(\mathbf{x}) \rightarrow \mathbb{R}^{3}$ depending on the mapping $\mathbf{x}$. We set $\mathbf{u}(\tau) \stackrel{\text { def }}{=} \mathbf{u}(\mathbf{x}+\tau \mathbf{x}): \Omega(\mathbf{x}+\tau \delta \mathbf{x}) \rightarrow \mathbb{R}^{3}$.

Definition 1 We say that the function $\mathbf{u}(\mathbf{x})$ admits a shape material derivative $\dot{\mathbf{u}}(\mathbf{x} ; \delta \mathbf{x})$ in the direction $\delta \mathbf{x}$ if the following limit exists,

$$
\dot{\mathbf{u}}(\mathbf{x} ; \delta \mathbf{x}) \stackrel{\text { def }}{=} \lim _{\tau \rightarrow 0} \frac{\mathbf{u}(\tau) \circ(\mathbf{x}+\tau \delta \mathbf{x})-\mathbf{u}(\mathbf{x}) \circ \mathbf{x}}{\tau}
$$

\section{Lemma 4 ([30], Section 2.31)}

$$
\mathrm{D}_{\mathbf{x}}\left[\int_{\Omega(\mathbf{x})} \mathbf{u} \cdot \mathbf{v} \mathrm{d} \mathbf{x}\right] \delta \mathbf{x}=\int_{\Omega(\mathbf{x})}[(\dot{\mathbf{u}}(\mathbf{x} ; \delta \mathbf{x}) \cdot \mathbf{v}+\mathbf{u} \cdot \dot{\mathbf{v}}(\mathbf{x} ; \delta \mathbf{x})+(\operatorname{div} \delta \mathbf{x}) \mathbf{u} \cdot \mathbf{v}] \mathrm{d} \mathbf{x}
$$


Let $U\left(\Omega_{0}\right)$ be a given abstract functional space of functions defined on $\Omega_{0}$, we define its transported image through $\mathbf{x}$ as follows,

$$
U(\Omega(\mathbf{x})) \stackrel{\text { def }}{=}\left\{\hat{\mathbf{u}} \circ \mathbf{x}^{-1}, \quad \hat{\mathbf{u}} \in U\left(\Omega_{0}\right)\right\},
$$

Lemma 5 For $((\mathbf{u}, p),(\mathbf{v}, q)) \in U(\Omega(\mathbf{x})) \times V(\Omega(\mathbf{x}))$, the following identities hold true,

$$
\begin{aligned}
\mathrm{D}_{\mathbf{x}}\left[\int_{\Omega(\mathbf{x})} \mathbf{u} \cdot \mathbf{v} \mathrm{d} \mathbf{x}\right] \delta \mathbf{x}= & \int_{\Omega(\mathbf{x})}(\operatorname{div} \delta \mathbf{x}) \mathbf{u} \cdot \mathbf{v} \mathrm{d} \mathbf{x} \\
\mathrm{D}_{\mathbf{x}}\left[\int_{\Omega(\mathbf{x})} q \operatorname{div} \mathbf{u} \mathrm{d} \mathbf{x}\right] \delta \mathbf{x}= & \int_{\Omega(\mathbf{x})} q \operatorname{div}\left\{\mathbf{u}\left[\operatorname{Idiv} \delta \mathbf{x}-(\nabla \delta \mathbf{x})^{\mathrm{T}}\right]\right\} \mathrm{d} \mathbf{x} \\
\mathrm{D}_{\mathbf{x}}\left[\int_{\Omega(\mathbf{x})} \sigma(\mathbf{u}, p): \nabla \mathbf{v} \mathrm{d} \mathbf{x}\right] \delta \mathbf{x}= & \int_{\Omega(\mathbf{x})} \sigma(\mathbf{u}, p)\left[\mathrm{I} \operatorname{div} \delta \mathbf{x}-(\nabla \delta \mathbf{x})^{\mathrm{T}}\right]: \nabla \mathbf{v} \mathrm{d} \mathbf{x} \\
& -\int_{\Omega(\mathbf{x})} \mu\left[\nabla \mathbf{u} \nabla \delta \mathbf{x}+(\nabla \delta \mathbf{x})^{\mathrm{T}}(\nabla \mathbf{u})^{\mathrm{T}}\right]: \nabla \mathbf{v} \mathrm{d} \mathbf{x}
\end{aligned}
$$

Proof. -In the special case where $((\mathbf{u}, p),(\mathbf{v}, q)) \in U(\Omega(\mathbf{x})) \times V(\Omega(\mathbf{x}))$, obviously we have

$$
((\dot{\mathbf{u}}(\mathbf{x} ; \delta \mathbf{x}), \dot{p}(\mathbf{x} ; \delta \mathbf{x})),(\dot{\mathbf{v}}(\mathbf{x} ; \delta \mathbf{x}), \dot{q}(\mathbf{x} ; \delta \mathbf{x}))=((0,0),(0,0))
$$

then using Lemma 4, we have

$$
\mathrm{D}_{\mathbf{x}}\left[\int_{\Omega(\mathbf{x})} \mathbf{u} \cdot \mathbf{v} \mathrm{d} \mathbf{x}\right] \delta \mathbf{x}=\int_{\Omega(\mathbf{x})}[(\operatorname{div} \delta \mathbf{x}) \mathbf{u} \cdot \mathbf{v}] \mathrm{d} \mathbf{x}
$$

The next results follows using the following identities,

$$
\begin{gathered}
\int_{\Omega(\mathbf{x})} q \operatorname{div} \mathbf{u} \mathrm{d} \mathbf{x}=\int_{\Omega_{0}} \hat{q} \operatorname{div}\left\{J \hat{\mathbf{u}} F^{-\mathrm{T}}\right\} \mathrm{d} \mathbf{x}_{0} \\
\int_{\Omega(\mathbf{x})} \nabla \mathbf{u}: \nabla \mathbf{v} \mathrm{d} \mathbf{x}=\int_{\Omega_{0}} J \nabla \hat{\mathbf{u}} F^{-1}: \nabla \hat{\mathbf{v}} F^{-1} \mathrm{~d} \mathbf{x}_{0}
\end{gathered}
$$

and the definition $\sigma(\mathbf{u}, p)=-p \mathrm{I}+\mu\left[\nabla \mathbf{u}+(\nabla \mathbf{u})^{\mathrm{T}}\right]$.

\section{References}

[1] S. Artlich and W. Mackens. Newton-coupling of fixed point iterations. In W. Hackbusch and G. Wittum, editors, Numerical treatment of coupled systems, volume 51 of Notes on Numerical Fluid Mechanics, pages 1-10, Wiesbaden, 1995. Vieweg.

INRIA 
[2] J.T. Batina. Unsteady Euler airfoil solutions using unstructured dynamic meshes. AIAA J., 28(8):1381-1388, 1990.

[3] M. Cervera, R. Codina, and M. Galindo. On the computational efficiency and implementation of block-iterative algorithms for nonlinear coupled problems. Engrg. Comput., 13(6):4-30, 1996.

[4] P.G. Ciarlet. Mathematical elasticity. Vol. I, volume 20 of Studies in Mathematics and its Applications. North-Holland Publishing Co., Amsterdam, 1988.

[5] S. Deparis. Axisymmetric flow in moving domains and fluid-structure interaction algorithms for blood flow modelling. PhD thesis, École Polytechnique Fédérale de Lausanne, 2004.

[6] S. Deparis, M.A. Fernández, and L. Formaggia. Acceleration of a fixed point algorithm for fluid-structure interaction using transpiration conditions. M2AN Math. Model. Numer. Anal., 37(4):601-616, 2003.

[7] S. Deparis, J.-F. Gerbeau, and X. Vasseur. Dynamic GMRES-based preconditioner for Newton or Quasi-Newton algorithms with application to fluid structure interaction. Submitted, 2004.

[8] J. Donea. An arbitrary lagrangian-eulerian finite element method for transient dynamic fluid-structure interactions. Comput. Methods Appl. Mech. Eng., 33:689-723, 1982.

[9] T. Fanion, M.A. Fernández, and P. Le Tallec. Deriving adequate formulations for fluidstructure interactions problems: from ALE to transpiration. Rév. Européenne Élém. Finis, 9(6-7):681-708, 2000. Also in A. Dervieux, editor, Fluid-Structure Interaction, chapter 3, Kogan Page Science, London, 2003.

[10] C. Farhat, M. Lesoinne, and P. Le Tallec. Load and motion transfer algorithms for fluid/structure interaction problems with non-matching discrete interfaces: momentum and energy conservation, optimal discretization and application to aeroelasticity. Comput. Methods Appl. Mech. Engrg., 157(1-2):95-114, 1998.

[11] M.A. Fernández and M. Moubachir. Sensitivity analysis for an incompressible aeroelastic system. Math. Models Methods Appl. Sci., 12(8):1109-1130, 2002.

[12] M.A. Fernández and M. Moubachir. An exact Block-Newton algorithm for solving fluid-structure interaction problems. C. R. Math. Acad. Sci. Paris, 336(8):681-686, 2003.

[13] M.A. Fernández and M. Moubachir. An exact Block-Newton algorithm for the solution of implicit time discretized coupled systems involved in fluid-structure interaction problems. In K.J. Bathe, editor, Second MIT Conference on Computational Fluid and Solid Mechanics, volume 2 of Computational Fluid and Solid Mechanics, pages 1337-1341, Cambridge (MA), June 17-20 2003. Elsevier. 
[14] L. Formaggia, J.-F. Gerbeau, F. Nobile, and A. Quarteroni. On the coupling of 3D and 1D Navier-Stokes equations for flow problems in compliant vessels. Comput. Methods Appl. Mech. Engrg., 191(6-7):561-582, 2001.

[15] L. Formaggia and F. Nobile. A stability analysis for the arbitrary Lagrangian Eulerian formulation with finite elements. East-West J. Numer. Math., 7(2):105-131, 1999.

[16] J.-F. Gerbeau and M. Vidrascu. A quasi-Newton algorithm based on a reduced model for fluid structure problems in blood flows. M2AN Math. Model. Numer. Anal., $37(4): 631-647,2003$.

[17] J.-F. Gerbeau, M. Vidrascu, and P. Frey. Fluid-structure interaction in blood flows on geometries coming from medical imaging. Technical Report 5052, INRIA, 2003.

[18] M.E. Gurtin. An introduction to continuum mechanics, volume 158 of Mathematics in Science and Engineering. Academic Press Inc., New York, 1981.

[19] M. Heil. An efficient solver for the fully-coupled solution of large-displacement fluidstructure interaction problems. Comput. Methods Appl. Mech. Engrg., 2003. In press.

[20] T.J.R. Hughes, W.K. Liu, and T.K. Zimmermann. Lagrangian-Eulerian finite element formulation for incompressible viscous flows. Comput. Methods Appl. Mech. Engrg., 29(3):329-349, 1981.

[21] P. Le Tallec. Numerical methods for nonlinear three-dimensional elasticity. In Handbook of numerical analysis, Vol. III, Handb. Numer. Anal., III, pages 465-622. NorthHolland, Amsterdam, 1994.

[22] P. Le Tallec and J. Mouro. Fluid structure interaction with large structural displacements. Comput. Methods Appl. Mech. Engrg., 190(24-25):3039-3067, 2001.

[23] H.G. Matthies and J. Steindorf. Partitioned but strongly coupled iteration schemes for nonlinear fluid-structure interaction. Computer \& Structures, 80:1991-1999, 2002.

[24] H.G. Matthies and J. Steindorf. Partitioned strong coupling algorithms for fluidstructure interaction. Computer \& Structures, 81:805-812, 2003.

[25] D.P. Mok, W.A. Wall, and E. Ramm. Accelerated iterative substructing schemes for instationary fluid-structure interaction. In Computational fluid and solid mechanics, Vol. 1, 2 (Cambridge, MA, 2001), pages 1325-1328. Elsevier, Amsterdam, 2001.

[26] H. Morand and R. Ohayon. Fluid-Structure Interaction. John Wiley \& Sons, Chichester, 1995.

[27] W.W. Nichols and M.F. O'Rourke. McDonald's Blood Flow in Arteries. Theoretical, experimental and clinical principles. Arnold, London, 1998. 
[28] F. Nobile. Numerical approximation of fluid-structure interaction problems with application to haemodynamics. PhD thesis, École Polytechnique Fédérale de Lausanne, 2001.

[29] S. Piperno, C. Farhat, and B. Larrouturou. Partitioned procedures for the transient solution of coupled aeroelastic problems. I. Model problem, theory and two-dimensional application. Comput. Methods Appl. Mech. Engrg., 124(1-2):79-112, 1995.

[30] J. Sokołowski and J.-P. Zolésio. Introduction to shape optimization, volume 16 of Springer Series in Computational Mathematics. Springer-Verlag, Berlin, 1992.

[31] T.E. Tezduyar. Finite element methods for fluid dynamics with moving boundaries and interfaces. Arch. Comput. Methods Engrg., 8:83-130, 2001.

[32] T.E. Tezduyar, M. Behr, and J. Liou. A new strategy for finite element computations involving moving boundaries and interfaces - the deforming-spatial-domain/space-time procedure. I. The concept and the preliminary numerical tests. Comput. Methods Appl. Mech. Engrg., 94(3):339-351, 1992.

[33] T.E. Tezduyar, M. Behr, S. Mittal, and J. Liou. A new strategy for finite element computations involving moving boundaries and interfaces - the deforming-spatialdomain/space-time procedure. II. Computation of free-surface flows, two-liquid flows, and flows with drifting cylinders. Comput. Methods Appl. Mech. Engrg., 94(3):353-371, 1992.

[34] P.D. Thomas and C.K. Lombard. Geometric conservation law and its application to flow computations on moving grids. AIAA J., 17(10):1030-1037, 1979. 


\section{Contents}

1 Introduction $\quad 3$

2 Mechanical problem $\quad 4$

$\begin{array}{lll}3 & \text { Continuous weak formulation } & 7\end{array}$

4 Time semi-discretised weak formulation $\quad 8$

4.1 An implicit coupling scheme . . . . . . . . . . . . . . . . 8

4.2 Abstract formulation . . . . . . . . . . . . . . . . . . 9

5 The Newton's algorithm $\quad 11$

6 Weak state operators derivatives $\quad 12$

6.1 Fluid operator derivatives . . . . . . . . . . . . . . . . . . 12

6.2 Solid operator derivatives $\ldots \ldots \ldots \ldots \ldots \ldots$

7 Detailed Newton's algorithm sub-steps $\quad 15$

$\begin{array}{lll}8 & \text { Numerical results } & 17\end{array}$

9 Conclusion $\quad 26$

$\begin{array}{ll}\text { A Shape derivative calculus } & \mathbf{2 6}\end{array}$ 


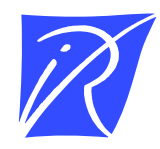

Unité de recherche INRIA Sophia Antipolis 2004, route des Lucioles - BP 93 - 06902 Sophia Antipolis Cedex (France)

Unité de recherche INRIA Lorraine : LORIA, Technopôle de Nancy-Brabois - Campus scientifique 615, rue du Jardin Botanique - BP 101 - 54602 Villers-lès-Nancy Cedex (France)

Unité de recherche INRIA Rennes : IRISA, Campus universitaire de Beaulieu - 35042 Rennes Cedex (France)

Unité de recherche INRIA Rhône-Alpes : 655, avenue de l'Europe - 38330 Montbonnot-St-Martin (France)

Unité de recherche INRIA Rocquencourt : Domaine de Voluceau - Rocquencourt - BP 105 - 78153 Le Chesnay Cedex (France)

Éditeur

INRIA - Domaine de Voluceau - Rocquencourt, BP 105 - 78153 Le Chesnay Cedex (France)

http://www.inria.fr

ISSN 0249-6399 